\title{
Copper-Mediated C-N Coupling of Arylsilanes with Nitrogen Nucleophiles
}

\author{
Johannes Morstein, Eric D. Kalkman, Chen Cheng, and John F. Hartwig* \\ Department of Chemistry, University of California, Berkeley, California 94720 \\ *jhartwig@berkeley.edu
}

\section{Supporting Information}

\section{Table of Contents}

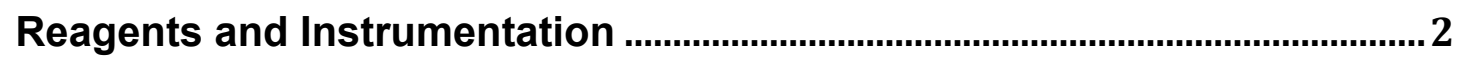

Reaction Profile of the formation of $3 \mathrm{~m}$ with and without $\mathrm{NaF}^{\mathrm{a}}$..................... 3

Evaluation of the $\mathrm{C}-\mathrm{N}$ coupling of arylsilanes with a series of silylgroups.

General Procedure for the Copper-Mediated C-N Coupling of Arylsilanes

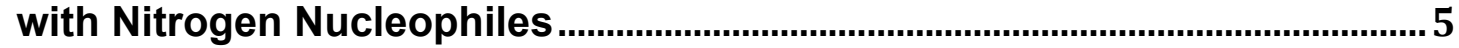

Procedure for the Copper-Mediated C-N Coupling of 1 with pyrrolidone

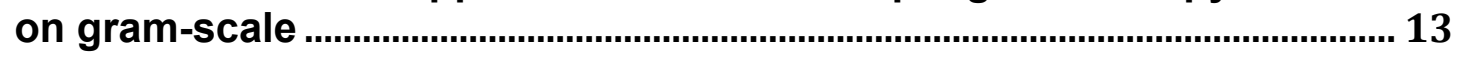

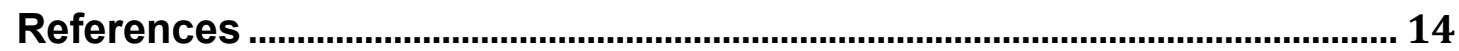

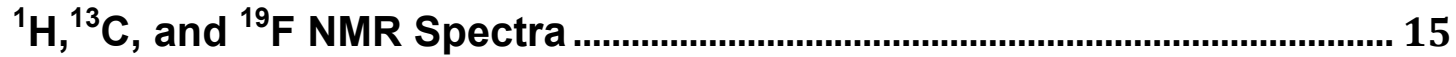




\section{Reagents and Instrumentation}

All air-sensitive manipulations were performed in a nitrogen-atmosphere glovebox. THF was purified by an Innovative Technology Pure-Solv solvent purification system.

Other solvents and reagents were purchased from commercial sources unless otherwise indicated. $[\operatorname{Ir}(\mathrm{cod}) \mathrm{OMe}]_{2}{ }^{1}$, arylsilanes ${ }^{2}$, and 2,4,7-trimethyl-1,10phenanthroline ${ }^{2 a}$ were synthesized according to literature procedures.

GC analyses were conducted on an Agilent 6890 GC equipped with an HP-5 column ( $25 \mathrm{~m} \times 0.20 \mathrm{~mm}$ ID $\times 0.33 \mathrm{~m}$ film) and an FID detector. Highresolution mass spectra were obtained via the Micro- Mass/Analytical Facility operated by the College of Chemistry, University of California, Berkeley. NMR spectra were acquired on Bruker AVQ-400, AVB-400, and AV-600 spectrometers. Chemical shifts were reported in ppm relative to residual solvent peaks $\left(\mathrm{CDCl}_{3}=7.26\right.$ ppm for ${ }^{1} \mathrm{H}$ and 77.16 ppm for ${ }^{13} \mathrm{C}$; DMSO $=2.50$ ppm for ${ }^{1} \mathrm{H}$ and 39.52 ppm for ${ }^{13} \mathrm{C}$ ). Coupling constants were reported in $\mathrm{Hz}$. Flash column chromatography was performed on a Teledyne ISCO CombiFlash $\AA$ Rf system. Preparative TLC was performed on Analtech $®$ Uniplate silica gel plates $(20 \mathrm{~cm} \times 20 \mathrm{~cm} \times 1 \mathrm{~mm})$. 
Reaction Profile of the formation of $3 \mathrm{~m}$ with and without $\mathrm{NaF}^{a}$

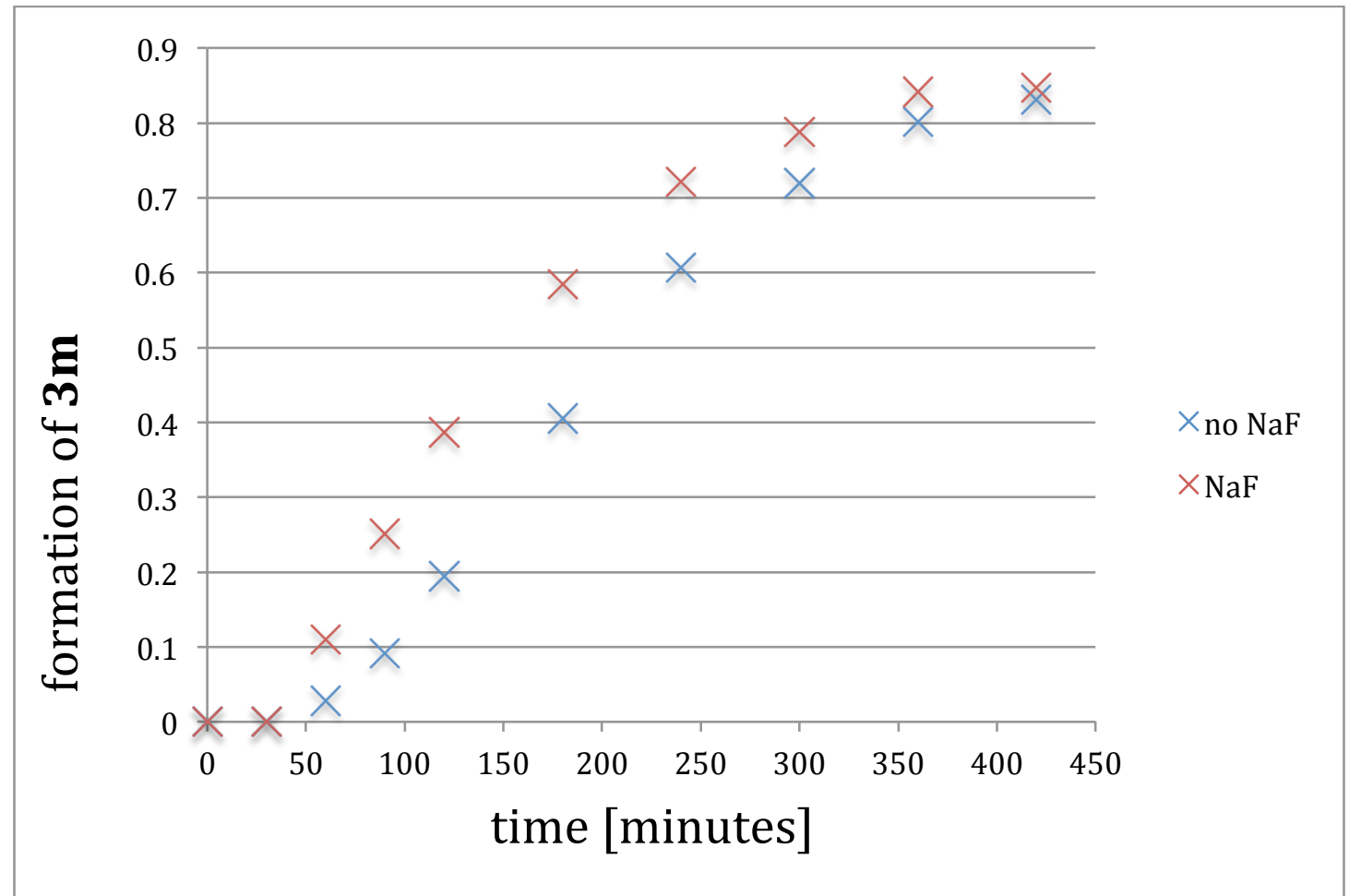

${ }^{\mathrm{a}}$ The formation of $3 \mathrm{~m}$ is monitored in the presence and absence of $\mathrm{NaF}$ by GCchromatography. (Factor of GC peak intensity calibration: starting material/product $=$ 1.56). Temperature: $80^{\circ} \mathrm{C}$. 


\section{Evaluation of the $\mathrm{C}-\mathrm{N}$ coupling of arylsilanes with different silyl-groups}

To test the applicability of the reported reaction conditions for to different silyl-groups, we evaluated the $\mathrm{C}-\mathrm{N}$ coupling of $6 \mathrm{a}-\mathbf{e}$ with pyrrolidone.

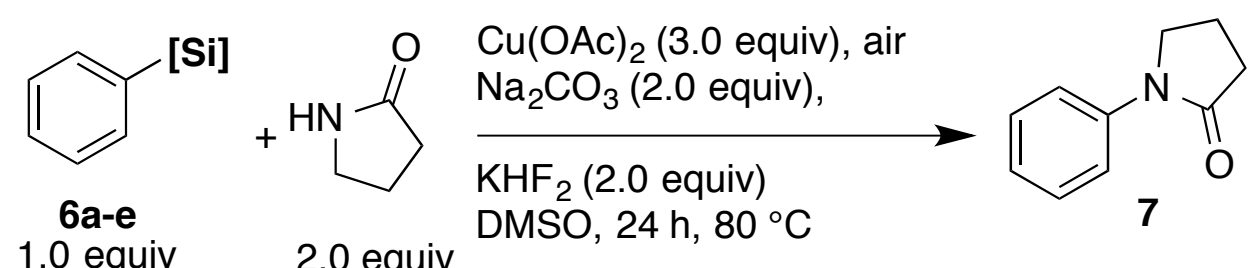

1.0 equiv 2.0 equiv

$$
\mathrm{Ph}-\mathrm{SiMe}_{3} \quad \mathrm{Ph}-\mathrm{SiPh}_{2} \mathrm{OH} \quad \mathrm{Ph}-\mathrm{SiMe}(\mathrm{OMe})_{2}
$$
$6 a, 1 \%$
$6 b, 60 \%$
$6 c, 27 \%$

$$
\mathrm{Ph}-\mathrm{SiMe}\left(\mathrm{OSiMe}_{3}\right)_{2} \quad \mathrm{Ph}-\mathrm{Si}(\mathrm{OMe})_{3}
$$

$6 d, 27 \%$

$6 e, 4 \%$

C-N coupling of arylsilanes with different silyl-groups and pyrrolidone. Yields by GC analysis.

The coupling of the silanol $\mathbf{6 b}$ gave a high yield of $\mathbf{7}$. The dialkoxysilanes $\mathbf{6 c}+\mathbf{d}$ both formed 7 in a moderate yield. Trace amounts of 7 were detected from the conversion of $6 a+e$. 


\section{General Procedure for the Copper-Mediated C-N Coupling of Arylsilanes with Nitrogen Nucleophiles}

To a $20 \mathrm{~mL}$ vial was added $\mathrm{Cu}(\mathrm{OAc})_{2}\left(0.90 \mathrm{mmol}, 3.0\right.$ equiv), $\mathrm{Na}_{2} \mathrm{CO}_{3}(0.60$ mmol, 2.0 equiv), NaF ( $0.60 \mathrm{mmol}, 2.0$ equiv), dry DMSO $(2.0 \mathrm{~mL})$, arylsilane ( $0.30 \mathrm{mmol}, 1.0$ equiv), and the nitrogen nucleophile $(0.60 \mathrm{mmol}, 2.0$ equiv) under air. The vial was sealed with a Teflon-lined cap, and the suspension was heated at $80{ }^{\circ} \mathrm{C}$ for $16 \mathrm{~h}$ with vigorous stirring. The suspension was allowed to cool to room temperature. Water $(5 \mathrm{~mL})$ was added to the reaction, and the resulting mixture was extracted with ethyl acetate $(3 \times 4 \mathrm{~mL})$. Extraction was performed in a $50 \mathrm{~mL}$ Falcon tube under centrifugation for convenient removal of insoluble components. The organic layer was washed with brine, dried over $\mathrm{MgSO}_{4}$, and filtered, and the solvent was evaporated under reduced pressure. The product was purified by flash column chromatography or preparative thin layer chromatography.

\section{Scheme 1, Entry 3b}<smiles>O=[N+]([O-])c1ccc(Nc2cc(Cl)cc(Cl)c2)cc1</smiles>

The reaction was performed according to the general procedure for the $\mathrm{C}-\mathrm{N}$ coupling of arylsilanes on a $0.30 \mathrm{mmol}$ scale. The crude mixture was purified by preparative TLC (3:7 ethyl acetate: hexanes) to give the product as a yellow solid $\left(60.6 \mathrm{mg}, 72 \%\right.$ yield). ${ }^{1} \mathrm{H}$ NMR $\left(600 \mathrm{MHz}\right.$, DMSO- $\left.d_{6}\right) \delta 9.49(\mathrm{~s}, 1 \mathrm{H}), 8.12$ (d, $J=8.5 \mathrm{~Hz}, 2 \mathrm{H}), 7.22-7.11(\mathrm{~m}, 5 \mathrm{H}) .{ }^{13} \mathrm{C}$ NMR $(151 \mathrm{MHz}$, DMSO) $\delta$ 148.68, 143.26, 139.48, 134.76, 125.95, 121.46, 117.30, 115.20. HR-ESI-MS calcd. for $\left[\mathrm{C}_{12} \mathrm{H}_{7} \mathrm{Cl}_{2} \mathrm{~N}_{2} \mathrm{O}_{2}(\mathrm{M}-\mathrm{H})\right]: 280.9890$, found: 280.9887 .

\section{Scheme 1, Entry 3c}<smiles>CC(=O)c1ccc(Nc2cc(Cl)cc(Cl)c2)cc1</smiles>

The reaction was performed according to the general procedure for the $\mathrm{C}-\mathrm{N}$ coupling of arylsilanes on a $0.30 \mathrm{mmol}$ scale. The crude mixture was purified by preparative TLC (1:1 ethyl acetate: hexanes) to give the product as a yellow solid (58.0 mg, 69\% yield). ${ }^{1} \mathrm{H}$ NMR (600 MHz, DMSO- $\left.d_{6}\right) \delta 9.13(\mathrm{~s}, 1 \mathrm{H}), 7.88$ $(\mathrm{d}, J=8.1 \mathrm{~Hz}, 2 \mathrm{H}), 7.15(\mathrm{~d}, J=8.1 \mathrm{~Hz}, 2 \mathrm{H}), 7.11(\mathrm{~s}, 2 \mathrm{H}), 7.03(\mathrm{~s}, 1 \mathrm{H}), 2.48(\mathrm{~s}$, $3 \mathrm{H}) .{ }^{13} \mathrm{C}$ NMR (151 MHz, DMSO) $\delta 195.66,146.27,144.48,134.65,130.29$, 129.49, 119.84, 115.96, 115.64, 26.17. HR-ESI-MS calcd. for $\left[\mathrm{C}_{14} \mathrm{H}_{12} \mathrm{Cl}_{2} \mathrm{NO}(\mathrm{M}+\mathrm{H})\right]^{+}: 280.0290$, found: 280.0289 .

\section{Scheme 1, Entry 3d}<smiles>COc1cc(Nc2cc(Cl)cc(Cl)c2)cc(C(F)(F)F)c1</smiles>

The reaction was performed according to the general procedure for the $\mathrm{C}-\mathrm{N}$ coupling of arylsilanes on a $0.30 \mathrm{mmol}$ scale. The crude 
mixture was purified by preparative TLC (1:1 ethyl acetate: hexanes) to give the product as a brown liquid $\left(90.2 \mathrm{mg}, 89 \%\right.$ yield). ${ }^{1} \mathrm{H}$ NMR $(600 \mathrm{MHz}$, Chloroform-d) $\delta 6.93(\mathrm{~s}, 1 \mathrm{H}), 6.92(\mathrm{~s}, 2 \mathrm{H}), 6.87(\mathrm{~s}, 1 \mathrm{H}), 6.78(\mathrm{~s}, 2 \mathrm{H}), 5.85(\mathrm{~s}$, $1 \mathrm{H}), 3.83(\mathrm{~s}, 3 \mathrm{H}) .{ }^{13} \mathrm{C} \mathrm{NMR}\left(151 \mathrm{MHz}, \mathrm{CDCl}_{3}\right) \delta 161.14,144.50,143.33$, 135.93, 133.09 (q, $J=32.0 \mathrm{~Hz}), 123.86$ (q, $J=272.5 \mathrm{~Hz}), 121.59,115.97$, 108.33, 107.72, 104.53, 55.74. ${ }^{19} \mathrm{~F} \mathrm{NMR}\left(376 \mathrm{MHz}, \mathrm{CDCl}_{3}\right) \delta-62.18$. HR-ESIMS calcd. for $\left[\mathrm{C}_{14} \mathrm{H}_{9} \mathrm{Cl}_{2} \mathrm{~F}_{3} \mathrm{NO}(\mathrm{M}-\mathrm{H})\right]: 334.0019$, found: 334.0018 .

\section{Scheme 1, Entry 3e}<smiles>COc1ccc(N(c2ccc(OC)cc2)c2cc(Cl)cc(Cl)c2)cc1</smiles>

The reaction was performed according to the general procedure for the $\mathrm{C}-\mathrm{N}$ coupling of arylsilanes on a $0.30 \mathrm{mmol}$ scale. The crude mixture was purified by preparative TLC (3:7 ethyl acetate: hexanes) to give the product as a colorless liquid $(60.3 \mathrm{mg}, 54 \%$ yield). ${ }^{1} \mathrm{H}$ NMR (600 MHz, Chloroform- $d$ ) $\delta 7.07$ (d, $J=8.8$ $\mathrm{Hz}, 4 \mathrm{H}), 6.87(\mathrm{~d}, \mathrm{~J}=8.8 \mathrm{~Hz}, 4 \mathrm{H}), 6.76(\mathrm{~s}, 1 \mathrm{H}), 6.69$ (s, $2 \mathrm{H}), 3.81(\mathrm{~s}, 6 \mathrm{H}) .{ }^{13} \mathrm{C}$ NMR $\left(151 \mathrm{MHz}, \mathrm{CDCl}_{3}\right) \delta$ 156.99, 150.91, 139.38, 135.22, 127.63, 119.22, 116.75, 115.17, 55.61. HRMS $\left[\mathrm{C}_{20} \mathrm{H}_{17} \mathrm{Cl}_{2} \mathrm{NO}_{2}(\mathrm{M})\right]$ calcd.: 373.0636 , found: 373.0628 .

\section{Scheme 1, Entry $3 f$}<smiles>Clc1ccc(Nc2cc(Cl)cc(Cl)c2)nc1</smiles>

The reaction was performed according to the general procedure for the $\mathrm{C}-\mathrm{N}$ coupling of arylsilanes on a $0.30 \mathrm{mmol}$ scale. The crude mixture was purified by preparative TLC (3:7 ethyl acetate: hexanes) to give the product as a colorless solid $(64.0 \mathrm{mg}, 78 \%$ yield). ${ }^{1} \mathrm{H}$ NMR $\left(400 \mathrm{MHz}, \mathrm{DMSO}-d_{6}\right) \delta 9.61(\mathrm{~s}, 1 \mathrm{H}), 8.23(\mathrm{~s}, 1 \mathrm{H}), 7.73(\mathrm{~s}, 2 \mathrm{H}), 7.67$ (d, $J=7.0 \mathrm{~Hz}, 1 \mathrm{H}), 6.98(\mathrm{~s}, 1 \mathrm{H}), 6.85$ (d, $J=8.8 \mathrm{~Hz}, 1 \mathrm{H}) .{ }^{13} \mathrm{C}$ NMR $(151 \mathrm{MHz}$, DMSO) $\delta 153.42,145.16,143.53,137.44,133.92,121.49,119.32,115.50$, 113.06. HRMS [ $\left.\mathrm{C}_{11} \mathrm{H}_{7} \mathrm{Cl}_{3} \mathrm{~N}_{2}(\mathrm{M})\right]$ calcd.: 271.9675 , found: 271.9665 .

\section{Scheme 1, Entry 3g}<smiles>Clc1cc(Cl)cc(Nc2ncccn2)c1</smiles>

The reaction was performed according to the general procedure for the $\mathrm{C}-\mathrm{N}$ coupling of arylsilanes on a 0.30 mmol scale. The crude mixture was purified by preparative TLC (1:1 ethyl acetate: hexanes $+5 \%$ acetone) to give the product as a colorless solid (47.0 $\mathrm{mg}, 65 \%$ yield). ${ }^{1} \mathrm{H}$ NMR (600 MHz, DMSO- $\left.d_{6}\right) \delta 10.05(\mathrm{~s}, 1 \mathrm{H}), 8.56(\mathrm{~s}, 2 \mathrm{H})$, 7.89 (s, 2H), 7.06 (s, 1H), 6.94 (s, 1H). ${ }^{13} \mathrm{C}$ NMR (151 MHz, DMSO) $\delta 159.37$, $158.13,143.01,133.86,120.00,116.27,113.58$. HR-ESI-MS calcd. for $\left[\mathrm{C}_{10} \mathrm{H}_{8} \mathrm{Cl}_{2} \mathrm{~N}_{3}(\mathrm{M}+\mathrm{H})\right]^{+}:$240.0090, found: 240.0089 . 
Scheme 1, Entry 3h<smiles>Clc1cc(Cl)cc(Nc2cnccn2)c1</smiles>

The reaction was performed according to the general procedure for the $\mathrm{C}-\mathrm{N}$ coupling of arylsilanes on a $0.30 \mathrm{mmol}$ scale. The crude mixture was purified by preparative TLC (1:1 ethyl acetate: hexanes) to give the product as a colorless solid (54.0 $\mathrm{mg}, 75 \%$ yield).

The TLC plate was pretreated with $\mathrm{Et}_{3} \mathrm{~N} .{ }^{1} \mathrm{H}$ NMR $\left(600 \mathrm{MHz}, \mathrm{DMSO}-d_{6}\right) \delta$ $9.88(\mathrm{~s}, 1 \mathrm{H}), 8.22(\mathrm{~d}, J=12.5 \mathrm{~Hz}, 2 \mathrm{H}), 8.02(\mathrm{~s}, 1 \mathrm{H}), 7.77(\mathrm{~s}, 2 \mathrm{H}), 7.07(\mathrm{~s}, 1 \mathrm{H})$. ${ }^{13} \mathrm{C}$ NMR (151 MHz, DMSO) $\delta 151.42,143.05,140.85,135.40,134.84$, 134.00, 119.98, 115.83. HR-ESI-MS calcd. for $\left[\mathrm{C}_{10} \mathrm{H}_{8} \mathrm{Cl}_{2} \mathrm{~N}_{3}(\mathrm{M}+\mathrm{H})\right]^{+}:$240.0090, found: 240.0088 .

\section{Scheme 1, Entry 3i}<smiles>Cn1ccc(Nc2cc(Cl)cc(Cl)c2)n1</smiles>

The reaction was performed according to the general procedure for the $\mathrm{C}-\mathrm{N}$ coupling of arylsilanes on a 0.30 $\mathrm{mmol}$ scale. The crude mixture was purified by preparative TLC (1:1 ethyl acetate: hexanes) to give the product as a colorless solid (42.0 mg, $58 \%$ yield). ${ }^{1} \mathrm{H}$

NMR (600 MHz, Chloroform-d) $\delta 7.26$ (s, 1H), 6.99 (s, 2H), 6.79 (s, 1H), 6.33 (s, 1H), $5.94(\mathrm{~s}, 1 \mathrm{H}), 3.83(\mathrm{~s}, 3 \mathrm{H}) .{ }^{13} \mathrm{C} \mathrm{NMR}\left(151 \mathrm{MHz}, \mathrm{CDCl}_{3}\right) \delta$ 149.65, 145.63, 135.45, 131.23, 119.25, 113.34, 96.03, 39.05. HRMS $\left[\mathrm{C}_{10} \mathrm{H}_{9} \mathrm{Cl}_{2} \mathrm{~N}_{3}(\mathrm{M})\right]$ calcd.: 241.0174, found: 241.0173 .

\section{Scheme 1, Entry 3j}<smiles>CC(=O)c1sccc1Nc1cc(Cl)cc(Cl)c1</smiles>

The reaction was performed according to the general procedure for the $\mathrm{C}-\mathrm{N}$ coupling of arylsilanes on a $0.30 \mathrm{mmol}$ scale. The crude mixture was purified by preparative TLC (3:7 ethyl acetate: hexanes) to give the product as a colorless solid (70.4 mg, 78\% yield). ${ }^{1} \mathrm{H}$ NMR (600 MHz, Chloroform-d) $\delta 8.84(\mathrm{~s}, 1 \mathrm{H}), 7.43$ (d, $J=5.4 \mathrm{~Hz}, 1 \mathrm{H}), 7.12(\mathrm{~d}, J=5.4 \mathrm{~Hz}, 1 \mathrm{H}), 7.02(\mathrm{~s}, 2 \mathrm{H}), 6.98(\mathrm{~s}, 1 \mathrm{H}), 3.87$ (s, $3 \mathrm{H}) .{ }^{13} \mathrm{C}$ NMR $\left(151 \mathrm{MHz}, \mathrm{CDCl}_{3}\right) \delta 165.05,149.23,143.60,135.72,132.19$, 122.36, 118.10, 117.38, 105.80, 51.77. HRMS $\left[\mathrm{C}_{12} \mathrm{H}_{9} \mathrm{Cl}_{2} \mathrm{NO}_{2} \mathrm{~S}(\mathrm{M})\right]$ calcd.: 300.9731 , found: 300.9734 .

\section{Scheme 1, Entry 3k}<smiles>COC(=O)c1cccn1-c1cc(Cl)cc(Cl)c1</smiles>

The reaction was performed according to the general procedure for the C-N coupling of arylsilanes on a 0.30 mmol scale. The crude mixture was purified by preparative TLC (3:7 ethyl acetate: hexanes) to give the product as a colorless solid $(51.0 \mathrm{mg}, 63 \%$ yield $)$.

${ }^{1} \mathrm{H}$ NMR (600 MHz, Chloroform-d) $\delta 8.84(\mathrm{~s}, 1 \mathrm{H}), 7.43(\mathrm{~d}, J=5.4 \mathrm{~Hz}, 1 \mathrm{H})$, $7.12(\mathrm{~d}, J=5.4 \mathrm{~Hz}, 1 \mathrm{H}), 7.02(\mathrm{~s}, 2 \mathrm{H}), 6.98(\mathrm{~s}, 1 \mathrm{H}), 3.87(\mathrm{~s}, 3 \mathrm{H}) .{ }^{13} \mathrm{C}$ NMR $(151$ $\left.\mathrm{MHz}, \mathrm{CDCl}_{3}\right) \delta 165.05,149.23,143.60,135.72,132.19,122.36,118.10$, 
117.38, 105.80, 51.77. HRMS $\left[\mathrm{C}_{12} \mathrm{H}_{9} \mathrm{Cl}_{2} \mathrm{NO}_{2}(\mathrm{M})\right]$ calcd.: 269.0010, found: 269.0007 .

\section{Scheme 1, Entry 3l}<smiles>CC(=O)Nc1cc(Cl)cc(Cl)c1</smiles>

The reaction was performed according to the general procedure for the C-N coupling of arylsilanes on a 0.30 mmol scale. The crude mixture was purified by preparative TLC (1:1 ethyl acetate: hexanes) to give the product as a colorless solid $\left(32.0 \mathrm{mg}, 53 \%\right.$ yield). ${ }^{1} \mathrm{H}$ NMR (400 MHz, DMSO) $\delta 10.26(\mathrm{~s}, 1 \mathrm{H}), 7.62(\mathrm{~s}, 2 \mathrm{H})$, 7.20 (s, 1H), 2.05 (s, 3H). ${ }^{13} \mathrm{C}$ NMR (151 MHz, DMSO) $\delta$ 169.01, 141.56, 134.02, 122.15, 116.97, 24.09. HRMS $\left[\mathrm{C}_{8} \mathrm{H}_{7} \mathrm{Cl}_{2} \mathrm{NO}(\mathrm{M})\right]$ calcd.: 202.9905, found: 202.9902.

\section{Scheme 1, Entry $3 \mathrm{~m}$}<smiles>O=C1CCCN1c1cc(Cl)cc(Cl)c1</smiles>

The reaction was performed according to the general procedure for the C-N coupling of arylsilanes on a 0.30 mmol scale. The crude mixture was purified by preparative TLC (1:1 ethyl acetate: hexanes) to give the product as a colorless solid $\left(53.0 \mathrm{mg}, 77 \%\right.$ yield). ${ }^{1} \mathrm{H}$ NMR $(600 \mathrm{MHz}$, Chloroform-d) $\delta 7.59$ (s, 2H), $7.10(\mathrm{~s}, 1 \mathrm{H}), 3.79(\mathrm{t}, J=7.0 \mathrm{~Hz}, 2 \mathrm{H}), 2.60$ (t, $J=$ $8.1 \mathrm{~Hz}, 2 \mathrm{H}), 2.16(\mathrm{p}, J=7.6 \mathrm{~Hz}, 2 \mathrm{H}) .{ }^{13} \mathrm{C} \mathrm{NMR}\left(151 \mathrm{MHz}, \mathrm{CDCl}_{3}\right) \delta 174.55$, $141.25,135.15,124.15,117.71,48.55,32.81,17.83$. HRMS $\left[\mathrm{C}_{10} \mathrm{H}_{9} \mathrm{Cl}_{2} \mathrm{NO}(\mathrm{M})\right]$ calcd.: 229.0061, found: 229.0061 .

\section{Scheme 1, Entry 3n}<smiles>Cc1ccc(S(=O)(=O)Nc2cc(Cl)cc(Cl)c2)cc1</smiles>

The reaction was performed according to the general procedure for the $\mathrm{C}-\mathrm{N}$ coupling of arylsilanes on a $0.30 \mathrm{mmol}$ scale. The crude mixture was purified by preparative TLC (1:1 ethyl acetate: hexanes) to give the product as a colorless solid (39.4 mg, 41\% yield). ${ }^{1} \mathrm{H}$ NMR $(600 \mathrm{MHz}$, Chloroform- $d$ ) $\delta 7.74$ (d, $J=7.8 \mathrm{~Hz}$, 2H), $7.46(\mathrm{~s}, 1 \mathrm{H}), 7.29$ (d, J = $7.7 \mathrm{~Hz}, 2 \mathrm{H}), 7.05(\mathrm{~s}, 1 \mathrm{H}), 7.02(\mathrm{~s}, 2 \mathrm{H}), 2.41$ (s, $3 \mathrm{H}) .{ }^{13} \mathrm{C}$ NMR $\left(151 \mathrm{MHz}, \mathrm{CDCl}_{3}\right) \delta 144.88,138.78,135.72,130.17,127.38$, 125.11, 118.68, 114.71, 21.74. HR-ESI-MS calcd. for $\left[\mathrm{C}_{13} \mathrm{H}_{10} \mathrm{Cl}_{2} \mathrm{NO}_{2} \mathrm{~S}(\mathrm{M}-\mathrm{H})\right]^{\text {: }}$ 313.9815, found: 313.9811 .

\section{Scheme 1, Entry 30}<smiles>FC(F)(F)C(F)(F)CNc1cc(Cl)cc(Cl)c1</smiles>

The reaction was performed according to the general procedure for the $\mathrm{C}-\mathrm{N}$ coupling of arylsilanes on a $0.30 \mathrm{mmol}$ scale. The crude mixture was purified by preparative TLC (2:8 ethyl acetate: hexanes) to give the product as a colorless liquid ( $35.7 \mathrm{mg}, 40 \%$ yield).

${ }^{1} \mathrm{H}$ NMR $(600 \mathrm{MHz}$, Chloroform-d) $\delta 6.79(\mathrm{~s}, 1 \mathrm{H}), 6.55(\mathrm{~s}, 2 \mathrm{H}), 4.03(\mathrm{~s}, 1 \mathrm{H})$, 
$3.79(\mathrm{t}, J=14.2 \mathrm{~Hz}, 2 \mathrm{H}) \cdot{ }^{13} \mathrm{C}$ NMR $\left(151 \mathrm{MHz}, \mathrm{CDCl}_{3}\right) \delta 148.16,135.90$, 119.24, $115.97(\mathrm{~m}), 111.67,43.86(\mathrm{t}, J=24.2 \mathrm{~Hz}), 31.05 .{ }^{19} \mathrm{~F}$ NMR $(376 \mathrm{MHz}$, Chloroform-d) $\delta-83.14,-121.05(\mathrm{t}, J=14.5 \mathrm{~Hz})$. HRMS $\left[\mathrm{C}_{9} \mathrm{H}_{6} \mathrm{Cl}_{2} \mathrm{~F}_{5} \mathrm{~N}(\mathrm{M})\right]$ calcd.: 291.9725, found: 291.9728.

\section{Scheme 1, Entry 3p}<smiles>CCOC(CNc1cc(Cl)cc(Cl)c1)OCC</smiles>

The reaction was performed according to the general procedure for the $\mathrm{C}-\mathrm{N}$ coupling of arylsilanes on a $0.30 \mathrm{mmol}$ scale. The crude mixture was purified by careful flash column chromatography (1:20 ethyl acetate: hexanes $\rightarrow 3: 17$ ethyl acetate: hexanes) to give the product as a colorless liquid $\left(34.8 \mathrm{mg}, 42 \%\right.$ yield). ${ }^{1} \mathrm{H}$ NMR (600 $\mathrm{MHz}$, Chloroform- $d$ ) $\delta 6.66(\mathrm{t}, J=1.7 \mathrm{~Hz}, 1 \mathrm{H}), 6.48(\mathrm{~d}, J=1.7 \mathrm{~Hz}, 2 \mathrm{H}), 4.64(\mathrm{t}$, $J=5.4 \mathrm{~Hz}, 1 \mathrm{H}), 3.72(\mathrm{dq}, J=9.3,7.1 \mathrm{~Hz}, 2 \mathrm{H}), 3.56(\mathrm{dq}, J=9.4,7.0 \mathrm{~Hz}, 2 \mathrm{H})$, $3.20(\mathrm{~d}, J=5.4 \mathrm{~Hz}, 2 \mathrm{H}), 1.24(\mathrm{t}, J=7.1 \mathrm{~Hz}, 6 \mathrm{H}) .{ }^{13} \mathrm{C} \mathrm{NMR}\left(151 \mathrm{MHz}, \mathrm{CDCl}_{3}\right)$ $\delta 149.75,135.60,117.38,111.33,100.60,62.69,46.18,15.48$. HRMS $\left[\mathrm{C}_{12} \mathrm{H}_{17} \mathrm{Cl}_{2} \mathrm{NO}_{2}(\mathrm{M})\right]$ calcd.: 227.0636 , found: 227.0636 .

\section{Scheme 2, Entry 5a}<smiles>COC(=O)c1cc(Cl)cc(N2CCCC2=O)c1</smiles>

The reaction was performed according to the general procedure for the C-N coupling of arylsilanes on a 0.30 mmol scale. The crude mixture was purified by preparative TLC (7:3 ethyl acetate: hexanes) to give the product as a colorless solid $\left(44.0 \mathrm{mg}, 58 \%\right.$ yield). ${ }^{1} \mathrm{H}$ NMR $(600 \mathrm{MHz}$, Chloroform-d) $\delta 8.14(\mathrm{~s}, 1 \mathrm{H}), 7.93(\mathrm{~s}, 1 \mathrm{H}), 7.75(\mathrm{~s}, 1 \mathrm{H}), 3.97-3.77(\mathrm{~m}, 5 \mathrm{H})$, $2.62(\mathrm{t}, J=7.4 \mathrm{~Hz}, 2 \mathrm{H}), 2.26-2.11(\mathrm{~m}, 2 \mathrm{H}) .{ }^{13} \mathrm{C} \mathrm{NMR}\left(151 \mathrm{MHz}, \mathrm{CDCl}_{3}\right) \delta$ $174.60,165.75,140.70,134.97,131.98,125.25,124.10,118.06,52.61$, 48.65, 32.77, 17.90. HRMS $\left[\mathrm{C}_{12} \mathrm{H}_{12} \mathrm{CINO}_{3}(\mathrm{M})\right]$ calcd.: 253.0506, found: 253.0507.

\section{Scheme 2, Entry 5b}<smiles>COc1cc(N2CCCC2=O)cc(C(F)(F)F)c1</smiles>

The reaction was performed according to the general procedure for the C-N coupling of arylsilanes on a 0.30 $\mathrm{mmol}$ scale. The crude mixture was purified by preparative TLC (7:3 ethyl acetate: hexanes) to give the product as a colorless liquid (45.3 mg, $58 \%$ yield). ${ }^{1} \mathrm{H}$ NMR $(600 \mathrm{MHz}$, Chloroform-d) $\delta 7.61(\mathrm{~s}, 1 \mathrm{H}), 7.34(\mathrm{~s}, 1 \mathrm{H}), 6.90(\mathrm{~s}, 1 \mathrm{H}), 3.94-3.73(\mathrm{~m}, 5 \mathrm{H})$, $2.62(\mathrm{t}, J=8.0 \mathrm{~Hz}, 2 \mathrm{H}), 2.17(\mathrm{p}, J=7.3 \mathrm{~Hz}, 2 \mathrm{H}) .{ }^{13} \mathrm{C} \mathrm{NMR}\left(151 \mathrm{MHz}, \mathrm{CDCl}_{3}\right)$ $\delta 174.71,160.29,141.26,132.00$ (q, $J=32.1,31.2 \mathrm{~Hz}), 123.94$ (d, $J=272.7$ $\mathrm{Hz}), 108.90,108.32,106.80,55.75,48.81,32.95,17.92 .{ }^{19} \mathrm{~F}$ NMR $(376 \mathrm{MHz}$, $\left.\mathrm{CDCl}_{3}\right) \delta$-65.33. HRMS $\left[\mathrm{C}_{12} \mathrm{H}_{12} \mathrm{~F}_{3} \mathrm{NO}_{2}(\mathrm{M})\right]$ calcd.: 259.0820 , found: 259.0819 . 


\section{Scheme 2, Entry 5c}<smiles>COS(=O)(=O)c1cc(Br)cc(N2CCCC2=O)c1</smiles>

The reaction was performed according to the general procedure for the $\mathrm{C}-\mathrm{N}$ coupling of arylsilanes on a 0.30 mmol scale. The crude mixture was purified by preparative TLC (ethyl acetate) to give the product as a colorless solid (82.1 mg, 86\% yield). ${ }^{1} \mathrm{H}$ NMR (600 MHz, Chloroform-d) $\delta$ $8.25(\mathrm{~s}, 1 \mathrm{H}), 8.01(\mathrm{~s}, 1 \mathrm{H}), 7.78(\mathrm{~s}, 1 \mathrm{H}), 3.87(\mathrm{~s}, 2 \mathrm{H}), 3.06(\mathrm{~s}, 3 \mathrm{H}), 2.63(\mathrm{~s}, 2 \mathrm{H})$, $2.19(\mathrm{~s}, 2 \mathrm{H}) .{ }^{13} \mathrm{C}$ NMR $\left(151 \mathrm{MHz}, \mathrm{CDCl}_{3}\right) \delta 174.82,142.50,141.55,127.02$, 125.28, 123.53, 116.11, 48.49, 44.42, 32.67, 17.78. HRMS $\left[\mathrm{C}_{11} \mathrm{H}_{12} \mathrm{BrNO}_{3}(\mathrm{M})\right]$ calcd.: 316.9721 , found: 316.9725 .

\section{Scheme 2, Entry 5d}

The reaction was performed according to the general<smiles>Cc1cc(C#N)cc(N2CCCC2=O)c1</smiles>
procedure for the C-N coupling of arylsilanes on a $0.30 \mathrm{mmol}$ scale. The crude mixture was purified by preparative TLC (7:3 ethyl acetate: hexanes) to give the product as a colorless solid (41.1 mg, 68\% yield). ${ }^{1} \mathrm{H}$ NMR $(600 \mathrm{MHz}$, Chloroform- $d$ ) $\delta 7.72$ (d, $J=10.1 \mathrm{~Hz}, 2 \mathrm{H}), 7.20$ (s, 1H), 3.83 $(\mathrm{t}, J=7.0 \mathrm{~Hz}, 2 \mathrm{H}), 2.61(\mathrm{t}, J=8.1 \mathrm{~Hz}, 2 \mathrm{H}), 2.37(\mathrm{~s}, 3 \mathrm{H}), 2.18(\mathrm{p}, J=7.6 \mathrm{~Hz}$, $2 \mathrm{H}) .{ }^{13} \mathrm{C}$ NMR $\left(151 \mathrm{MHz}, \mathrm{CDCl}_{3}\right) \delta 174.61,140.13,128.16,124.47,119.95$, 118.85, 112.60, 48.52, 32.72, 21.50, 17.90. HRMS $\left[\mathrm{C}_{12} \mathrm{H}_{12} \mathrm{~N}_{2} \mathrm{O}(\mathrm{M})\right]$ calcd.: 200.0950, found: 200.0950 .

\section{Scheme 2, Entry 5e}<smiles>CCOC(=O)c1cc(N2CCCC2=O)cc(C(F)(F)F)c1</smiles>

The reaction was performed according to the general procedure for the $\mathrm{C}-\mathrm{N}$ coupling of arylsilanes on a 0.30 mmol scale. The crude mixture was purified by preparative TLC (2:8 acetone: ethyl acetate) to give the product as a colorless liquid (77.3 mg, 78\% yield). ${ }^{1} \mathrm{H}$ NMR $(400 \mathrm{MHz}$, Chloroform- $d$ ) $\delta 7.90(\mathrm{~d}, J=4.5 \mathrm{~Hz}, 2 \mathrm{H}), 7.39(\mathrm{~s}, 1 \mathrm{H}), 3.88(\mathrm{t}, J=6.7 \mathrm{~Hz}, 2 \mathrm{H})$, 3.54 (s, 2H), 3.25 (s, 2H), $2.68-2.57(\mathrm{~m}, 2 \mathrm{H}), 2.19$ (p, J=7.3 Hz, 2H), 1.19 $(\mathrm{d}, J=40.0 \mathrm{~Hz}, 6 \mathrm{H}) .{ }^{13} \mathrm{C} \mathrm{NMR}\left(151 \mathrm{MHz}, \mathrm{CDCl}_{3}\right) \delta 174.68,169.44,140.22$, 138.70, 131.62 (q, $J=32.6 \mathrm{~Hz}), 123.65$ (d, $J=273.2 \mathrm{~Hz}), 120.58,118.91$, 116.64, 48.58, 43.59, 39.68, 32.73, 17.94, 14.27, 12.94. ${ }^{19} \mathrm{~F}$ NMR (376 MHz, $\left.\mathrm{CDCl}_{3}\right) \quad \delta$-62.78. HRMS $\left[\mathrm{C}_{16} \mathrm{H}_{19} \mathrm{~F}_{3} \mathrm{~N}_{2} \mathrm{O}_{2}(\mathrm{M})\right]$ calcd.: 328.1399, found: 328.1398.

\section{Scheme 2, Entry $5 f$}<smiles>COc1ccc(N2CCCC2=O)cc1C(F)(F)F</smiles>

The reaction was performed according to the general procedure for the $\mathrm{C}-\mathrm{N}$ coupling of arylsilanes on a 0.30 mmol scale. The crude mixture was purified by preparative TLC (3:7 hexanes: ethyl acetate) to give the product as a colorless liquid (48.9 mg, 63\% yield). ${ }^{1} \mathrm{H}$

NMR (600 MHz, Chloroform-d) $\delta 7.82$ (d, J = 8.1 Hz, 1H), 7.71 (s, 1H), 6.99 
(d, $J=8.5 \mathrm{~Hz}, 1 \mathrm{H}), 3.91-3.74(\mathrm{~m}, 5 \mathrm{H}), 2.59(\mathrm{t}, J=7.2 \mathrm{~Hz}, 2 \mathrm{H}), 2.21-2.11$ (m, 2H). ${ }^{13} \mathrm{C}$ NMR $\left(151 \mathrm{MHz}, \mathrm{CDCl}_{3}\right) \delta 174.26,154.28,132.29,125.37$, 123.45 (q, $J=272.8 \mathrm{~Hz}), 119.10(\mathrm{q}, J=5.5 \mathrm{~Hz}), 118.90-118.42(\mathrm{~m}), 112.60$, 56.30, 48.98, 32.45, 17.98. ${ }^{19} \mathrm{~F} \mathrm{NMR}\left(376 \mathrm{MHz}, \mathrm{CDCl}_{3}\right) \delta-61.65$. HR-ESI-MS calcd. for $\left[\mathrm{C}_{12} \mathrm{H}_{12} \mathrm{~F}_{3} \mathrm{NNaO}_{2}(\mathrm{M}+\mathrm{Na})^{+}\right]$: 282.0712, found: 282.0708 .

\section{Scheme 2, Entry 5g}<smiles>COC(=O)c1cc2ccc(N3CCCC3=O)cc2cc1OC</smiles>
The reaction was performed according to the general procedure for the $\mathrm{C}-\mathrm{N}$ coupling of arylsilanes on a $0.30 \mathrm{mmol}$ scale. The crude mixture was purified by flash column chromatography (1:1 ethyl acetate: hexanes) to give the product as a colorless solid ( $58.7 \mathrm{mg}, 60 \%$ yield). ${ }^{1} \mathrm{H}$ NMR $(600 \mathrm{MHz}$, Chloroform- $d$ ) $\delta 8.23$ (s, 1H), 8.19 (dd, $J=9.0,2.2 \mathrm{~Hz}, 1 \mathrm{H}), 8.16(\mathrm{~s}, 1 \mathrm{H}), 7.92(\mathrm{~d}, J=2.0 \mathrm{~Hz}, 1 \mathrm{H})$, 7.90 (d, $J=9.1 \mathrm{~Hz}, 1 \mathrm{H}$ ), 3.98 (t, $J=7.0 \mathrm{~Hz}, 2 \mathrm{H}$ ), 3.95 (d, $J=3.4 \mathrm{~Hz}, 6 \mathrm{H}), 2.70$ - $2.65(\mathrm{~m}, 2 \mathrm{H}), 2.23(\mathrm{p}, J=7.6 \mathrm{~Hz}, 2 \mathrm{H}) \cdot{ }^{13} \mathrm{C}$ NMR $\left(151 \mathrm{MHz}, \mathrm{CDCl}_{3}\right) \delta$ $174.80,168.46,168.04,139.86,133.98,130.26,130.12,129.69,129.53$, 129.47, 127.36, 122.10, 116.52, 52.83, 52.77, 48.86, 32.99, 18.09. HR-ESIMS calcd. for $\left[\mathrm{C}_{18} \mathrm{H}_{17} \mathrm{NNaO}_{5}(\mathrm{M}+\mathrm{Na})^{+}\right]$]: 350.0999 , found: 350.1006 .

\section{Scheme 2, Entry $5 \mathrm{~h}$}<smiles>Cc1ccc(N2CCCC2=O)cc1C</smiles>

The reaction was performed according to the general procedure for the C-N coupling of arylsilanes on a $0.30 \mathrm{mmol}$ scale. The crude mixture was purified by preparative TLC (1:1 ethyl acetate: hexanes) to give the product as a colorless solid (21.1 mg, 37\% yield). ${ }^{1} \mathrm{H}$ NMR $(600 \mathrm{MHz}$, Chloroform- $d$ ) $\delta 7.39(\mathrm{~s}, 0 \mathrm{H}), 7.28(\mathrm{~d}, J=7.2 \mathrm{~Hz}, 1 \mathrm{H}), 7.11(\mathrm{~d}, J=7.6 \mathrm{~Hz}, \mathrm{OH}), 3.83(\mathrm{~s}, 1 \mathrm{H})$, $2.60(\mathrm{t}, J=7.3 \mathrm{~Hz}, 1 \mathrm{H}), 2.26(\mathrm{~s}, 1 \mathrm{H}), 2.23(\mathrm{~s}, 1 \mathrm{H}), 2.18-2.09(\mathrm{~m}, 1 \mathrm{H}) \cdot{ }^{13} \mathrm{C}$ NMR $\left(151 \mathrm{MHz}, \mathrm{CDCl}_{3}\right) \delta 174.10,137.08,136.99,133.04,129.78,121.68$, 117.77, 49.08, 32.61, 19.96, 19.14, 18.04. HR-ESI-MS calcd. for $\left[\mathrm{C}_{12} \mathrm{H}_{15} \mathrm{NNaO}(\mathrm{M}+\mathrm{Na})^{+}\right]: 212.1046$, found: 212.1050 .

\section{Scheme 2, Entry $5 i^{3}$}<smiles>COc1ccc(N2CCCC2=O)cc1OC</smiles>

The reaction was performed according to the general procedure for the $\mathrm{C}-\mathrm{N}$ coupling of arylsilanes on a 0.30 mmol scale at $100^{\circ} \mathrm{C}$ for $2 \mathrm{~d}$. The crude mixture was purified by column chromatography (ethyl acetate) to give the product as a light yellow solid $(9.1 \mathrm{mg}, 14 \%$ yield $) .{ }^{1} \mathrm{H}$

NMR (500 MHz, Chloroform-d) $\delta 7.50$ (s, 1H), 6.84 (s, 2H), 3.89 (s, 3H), 3.86 (s, 3H), $3.83(\mathrm{t}, J=7.0 \mathrm{~Hz}, 2 \mathrm{H}), 2.60(\mathrm{t}, J=8.1 \mathrm{~Hz}, 2 \mathrm{H}), 2.15(\mathrm{p}, J=7.5 \mathrm{~Hz}$, 2H). ${ }^{13} \mathrm{C}$ NMR $\left(126 \mathrm{MHz}, \mathrm{CDCl}_{3}\right) \delta 174.22,148.98,146.20,133.16,111.91$, $111.01,105.33,56.06,49.42,32.79,18.07$. 
Scheme 2, Entry 5j<smiles>Cc1ncc(Nc2ccc(Cl)cn2)cn1</smiles>

The reaction was performed according to the general procedure for the $\mathrm{C}-\mathrm{N}$ coupling of arylsilanes on a 0.30 $\mathrm{mmol}$ scale. The crude mixture was purified by preparative TLC (3:7 ethyl acetate: hexanes) to give the product as a colorless solid $\left(39.4 \mathrm{mg}, 60 \%\right.$ yield). ${ }^{1} \mathrm{H}$ NMR $(600 \mathrm{MHz}$, DMSO-d $\left.d_{6}\right) \delta 9.47(\mathrm{~s}, 1 \mathrm{H}), 8.96(\mathrm{~s}, 2 \mathrm{H}), 8.19(\mathrm{~s}, 1 \mathrm{H}), 7.69(\mathrm{~d}, J=8.9 \mathrm{~Hz}, 1 \mathrm{H})$, $6.87(\mathrm{~d}, J=8.9 \mathrm{~Hz}, 1 \mathrm{H}), 2.53(\mathrm{~s}, 3 \mathrm{H}) .{ }^{13} \mathrm{C}$ NMR (151 MHz, DMSO) $\delta 159.13$, $153.64,145.24,137.53,133.73,120.98,112.41,24.74$. ES- calcd. for $\left[\mathrm{C}_{10} \mathrm{H}_{8} \mathrm{CIN}_{4}(\mathrm{M}-\mathrm{H})^{-}\right]:$219.0437, found: 219.0439 . 


\section{Procedure for the Copper-Mediated C-N Coupling of 1 with pyrrolidone on gram-scale}

To a suspension of $\mathrm{Cu}(\mathrm{OAc})_{2}(2.50 \mathrm{~g}, 20.4 \mathrm{mmol}), \mathrm{Na}_{2} \mathrm{CO}_{3}(1.44 \mathrm{~g}, 13.6$ $\mathrm{mmol}), \mathrm{NaF}(0.571 \mathrm{~g}, 13.6 \mathrm{mmol})$ in dry DMSO $(40.0 \mathrm{~mL})$ were added $1(2.50$ $\mathrm{g}, 6.80 \mathrm{mmol})$ and pyrrolidone $(1.16 \mathrm{~g}, 13.6 \mathrm{mmol})$. The suspension was heated at $80^{\circ} \mathrm{C}$ for $16 \mathrm{~h}$ with vigorous stirring. The suspension was allowed to cool to room temperature. Water $(50 \mathrm{~mL})$ was added to the reaction, and the resulting mixture was extracted with ethyl acetate $(3 \times 40 \mathrm{~mL})$. The organic layer was washed with brine, dried over $\mathrm{MgSO}_{4}$, filtered, and the solvent was evaporated under reduced pressure. The crude mixture was purified by preparative TLC (1:1 ethyl acetate: hexanes) to give the product as a colorless solid ( $1.19 \mathrm{~g}, 76 \%$ yield). 


\section{References}

(1) Tajuddin, H.; Harrisson, P.; Bitterlich, B.; Collings, J. C.; Sim, N.; Batsanov, A. S.; Cheung, M. S.; Kawamorita, S.; Maxwell, A. C.; Shukla, L.; Morris, J.; Lin, Z.; Marder, T. B.; Steel, P. G. Chem. Sci. 2012, 3, 3505.

(2) (a) Cheng, C.; Hartwig, J. F. J. Am. Chem. Soc. 2015, 137, 592; (b) Cheng, C.; Hartwig, J. F. Science 2014, 343, 853; (c) Morstein, J.; Hou, H.; Cheng, C.; Hartwig, J. F. Angew. Chem. Int. Ed. 2016, 55, 8054.

(3) Bathini, T.; Rawat, V. S.; Sreedhar, B. Synlett 2015, 26, 1348. 
${ }^{1} \mathrm{H},{ }^{13} \mathrm{C}$, and ${ }^{19} \mathrm{~F}$ NMR Spectra 
Scheme 1, Entry 3b

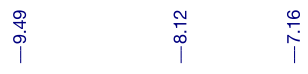<smiles>O=[N+]([O-])c1ccc(Nc2cc(Cl)cc(Cl)c2)cc1</smiles>

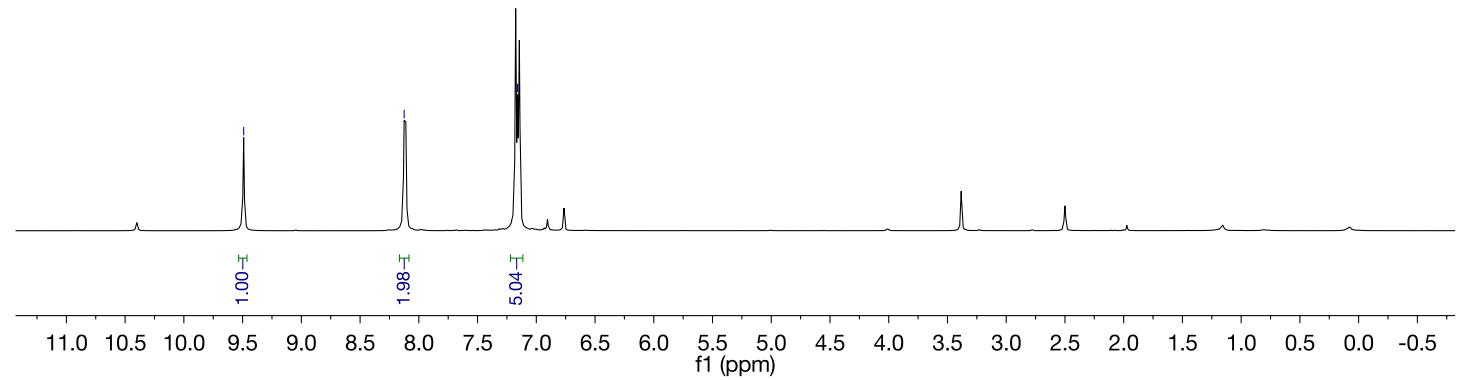

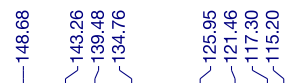<smiles>O=[N+]([O-])c1ccc(Nc2cc(Cl)cc(Cl)c2)cc1</smiles>

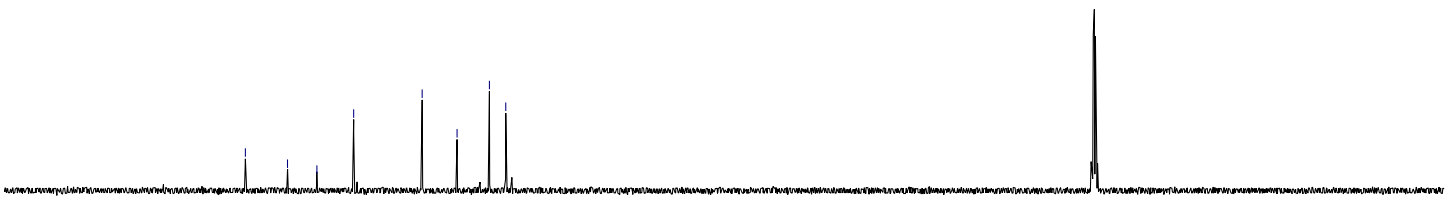

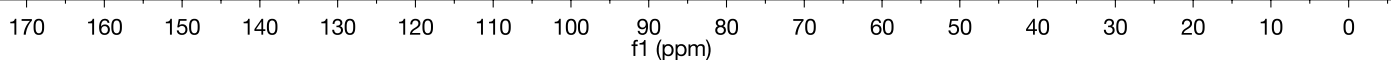


Scheme 1, Entry 3c

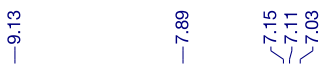<smiles>CC(=O)c1ccc(Nc2cc(Cl)cc(Cl)c2)cc1</smiles>

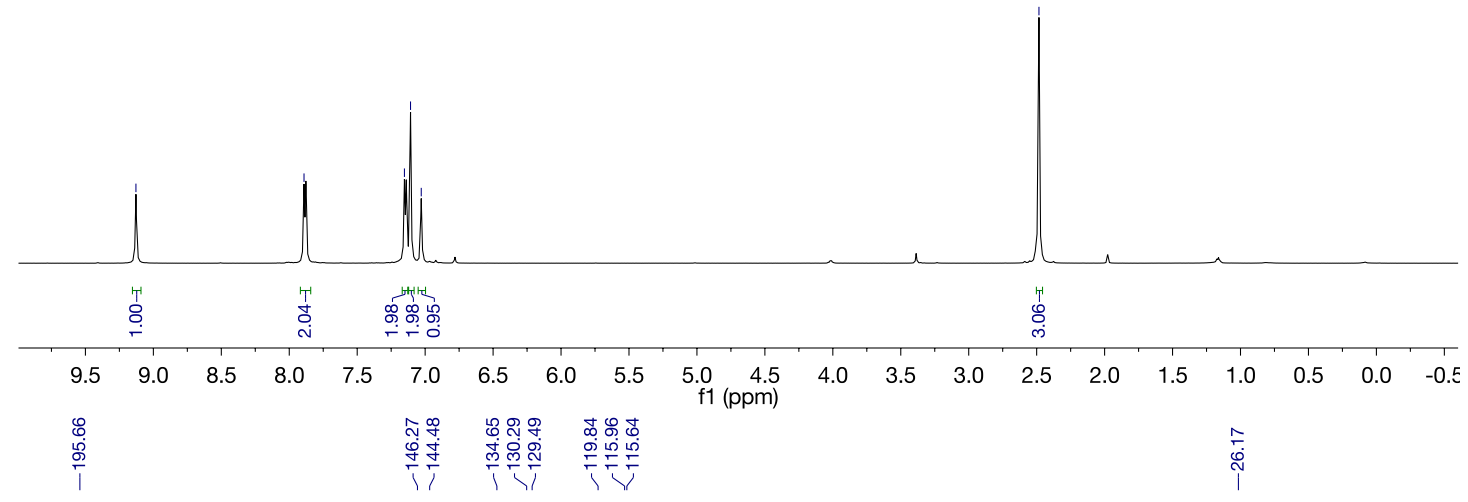<smiles>CC(=O)c1ccc(Nc2cc(Cl)cc(Cl)c2)cc1</smiles>

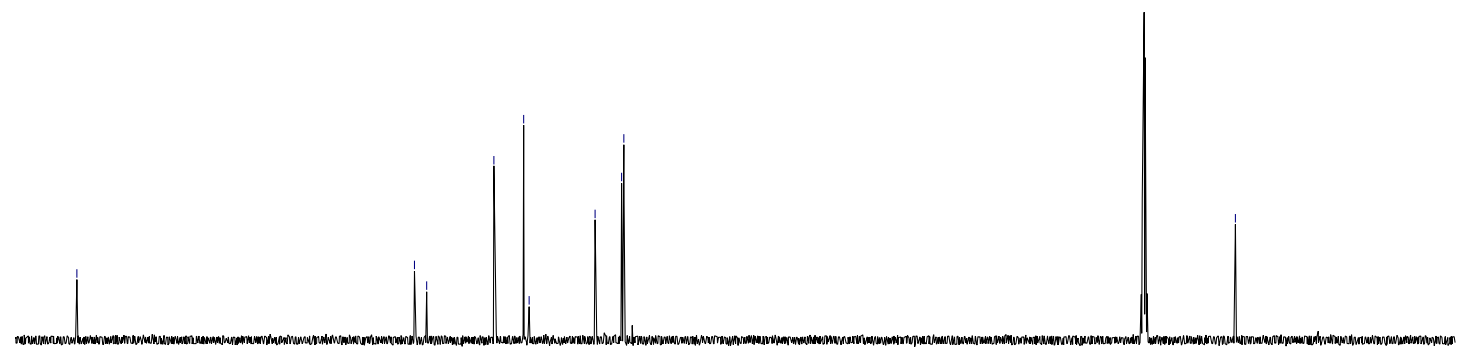

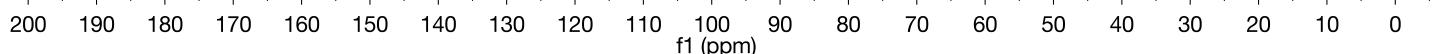


Scheme 1, Entry 3d
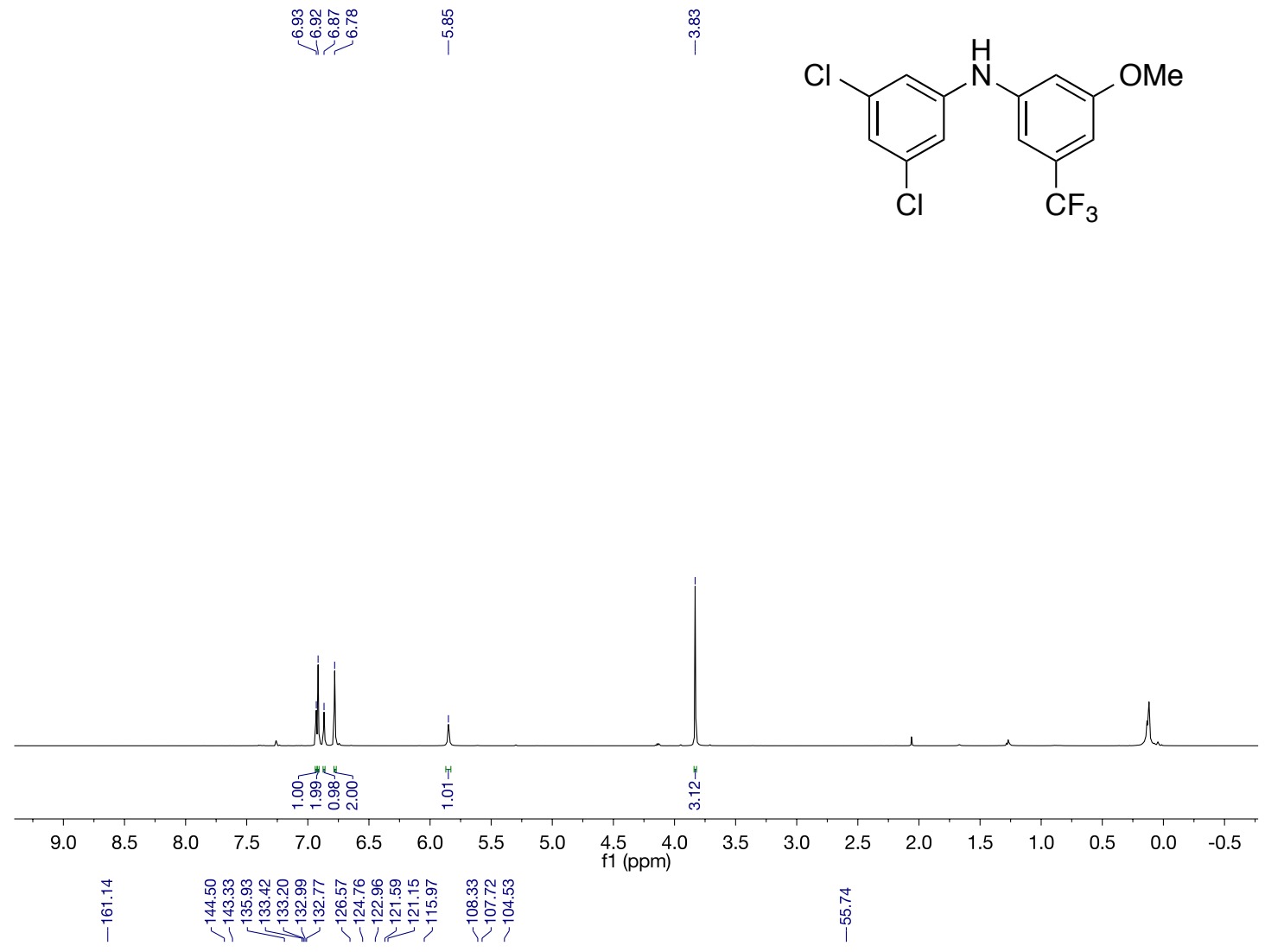<smiles>COc1cc(Nc2cc(Cl)cc(Cl)c2)cc(C(F)(F)F)c1</smiles>

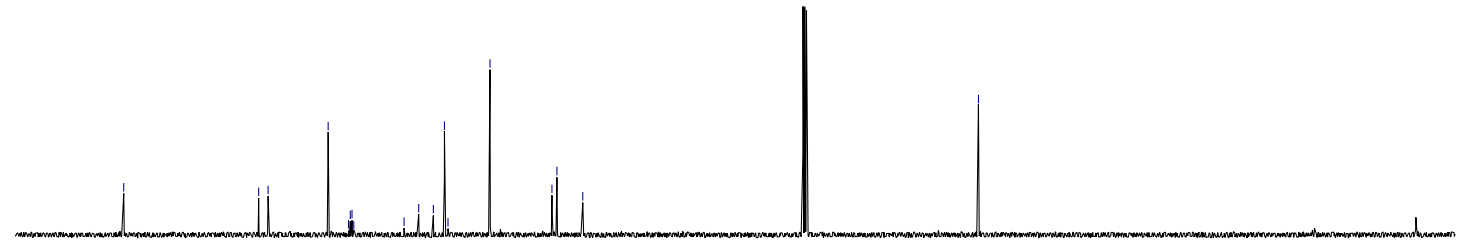

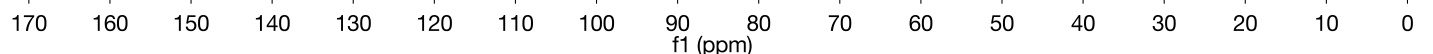


<smiles>COc1cc(Nc2cc(Cl)cc(Cl)c2)cc(C(F)(F)F)c1</smiles>

$\begin{array}{lllllllllllllllllllllllll}20 & 110 & 100 & 90 & 80 & 70 & 60 & 50 & 40 & 30 & 20 & 10 & 0 & 0 & 10 & -20 & -30 & -40 & -50 & -60 & -70 & -80 & -90 & -100 & -110\end{array}$ 
Scheme 1, Entry 3e

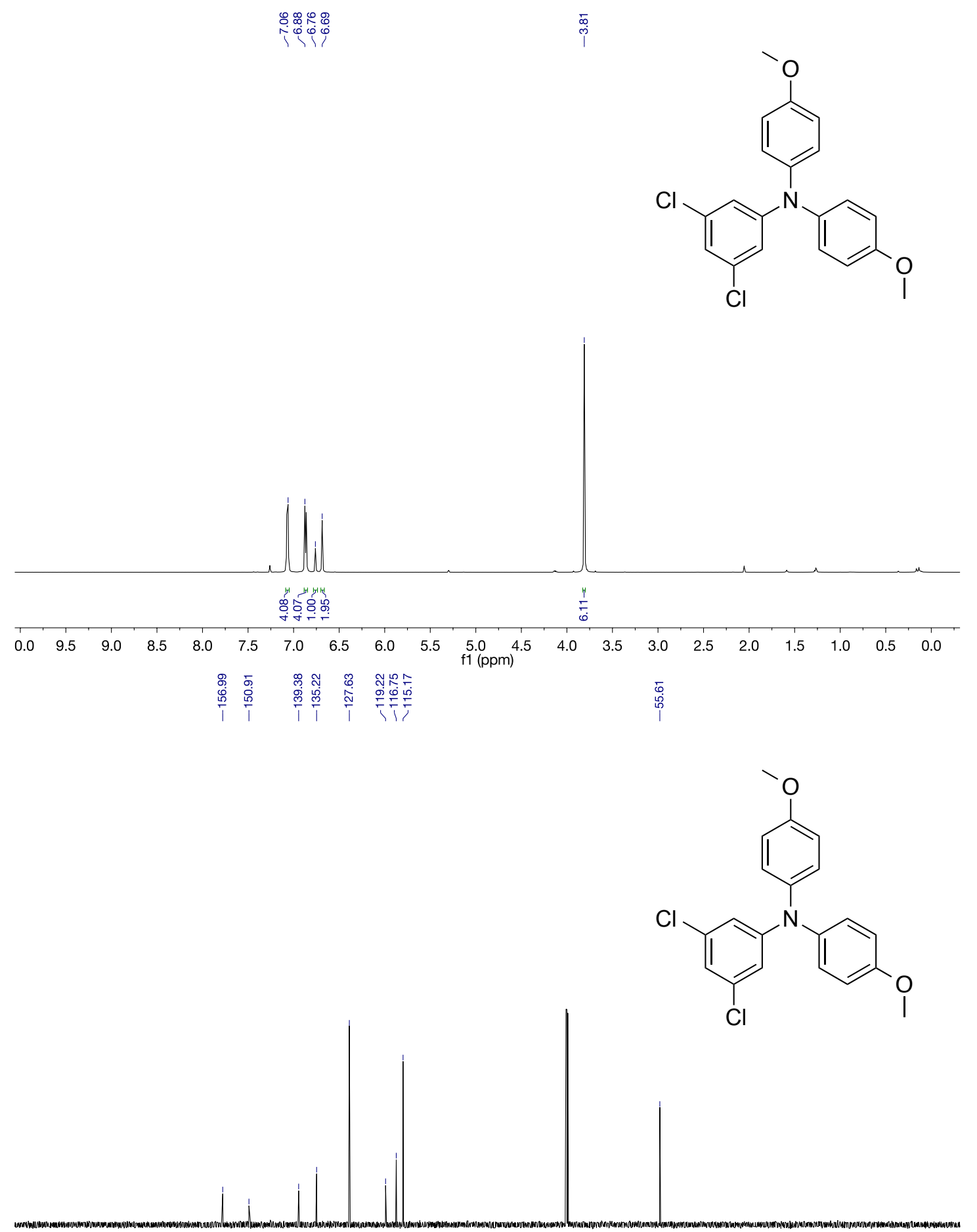

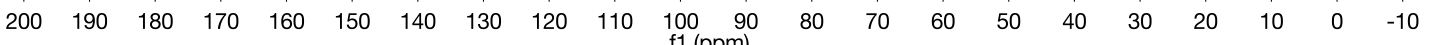


Scheme 1, Entry $3 f$

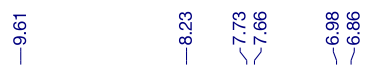<smiles>Clc1ccc(Nc2cc(Cl)cc(Cl)c2)nc1</smiles>

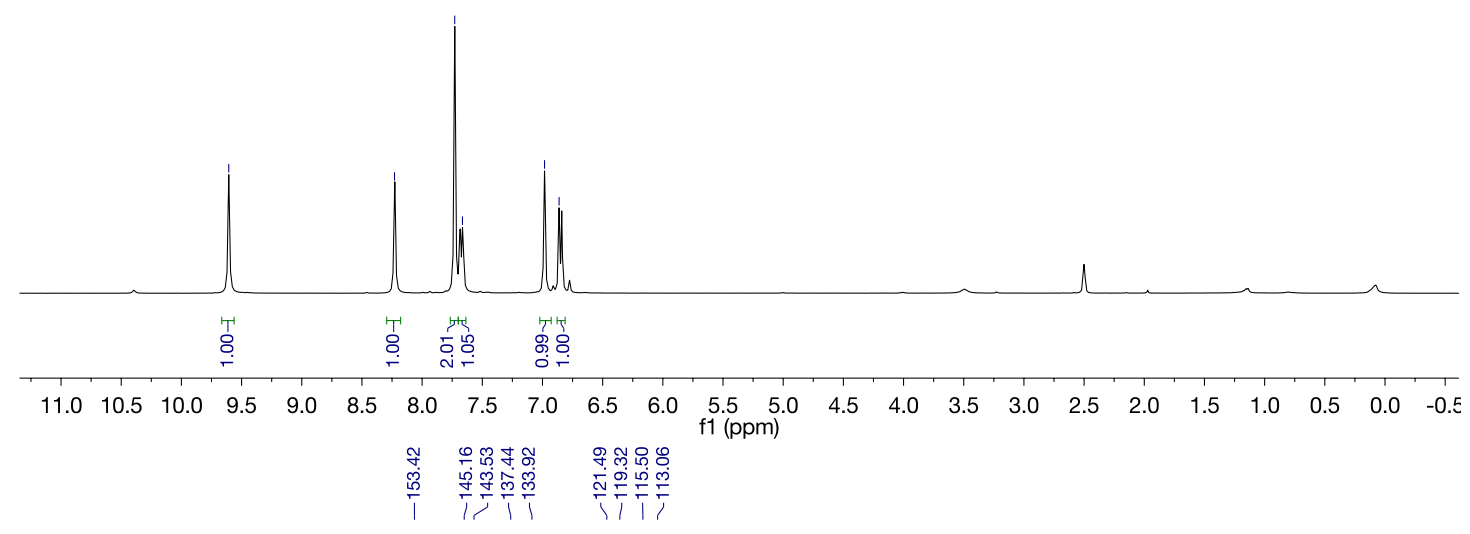<smiles>Clc1ccc(Nc2cc(Cl)cc(Cl)c2)nc1</smiles>

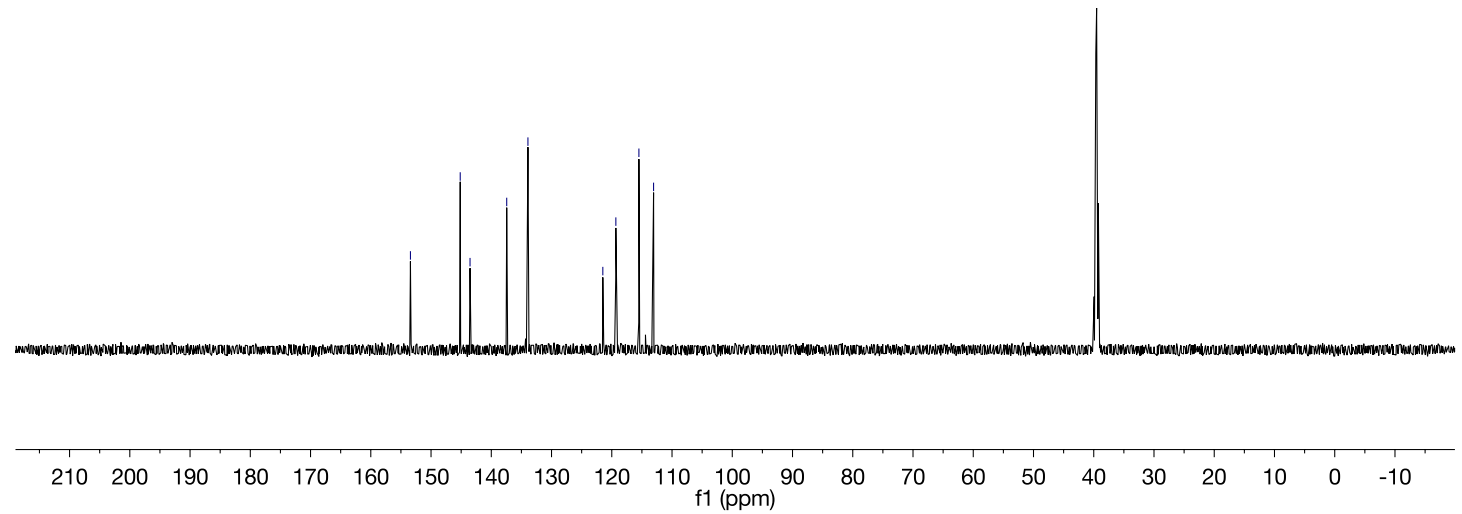


Scheme 1, Entry 3g

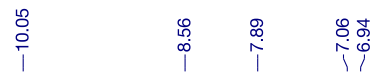

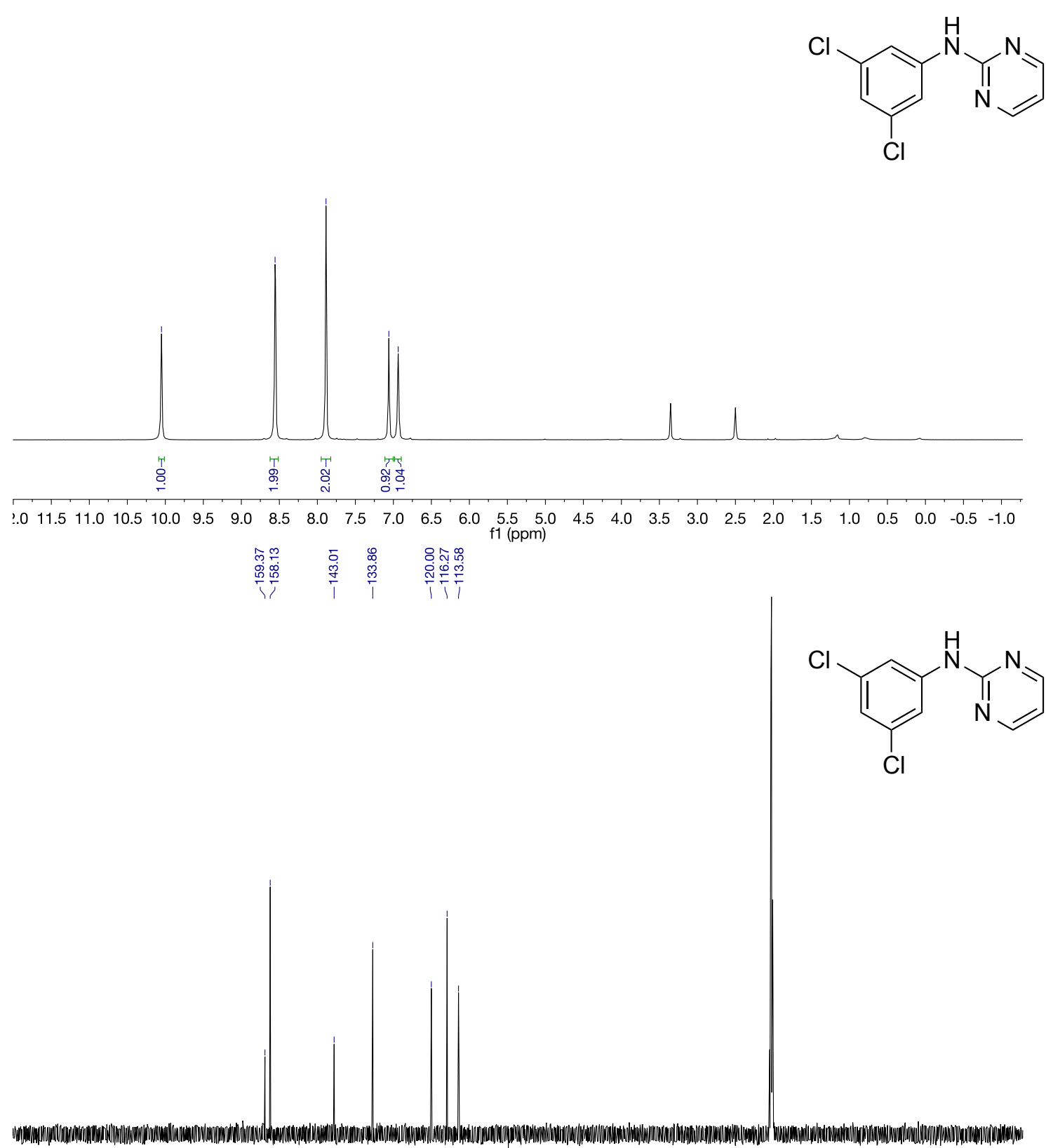

$\begin{array}{lllllllllllllllllllllll}210 & 200 & 190 & 180 & 170 & 160 & 150 & 140 & 130 & 120 & 110 & 100 & 90 & 80 & 70 & 60 & 50 & 40 & 30 & 20 & 10 & 0 & -10\end{array}$ 
Scheme 1, Entry $3 \mathrm{~h}$

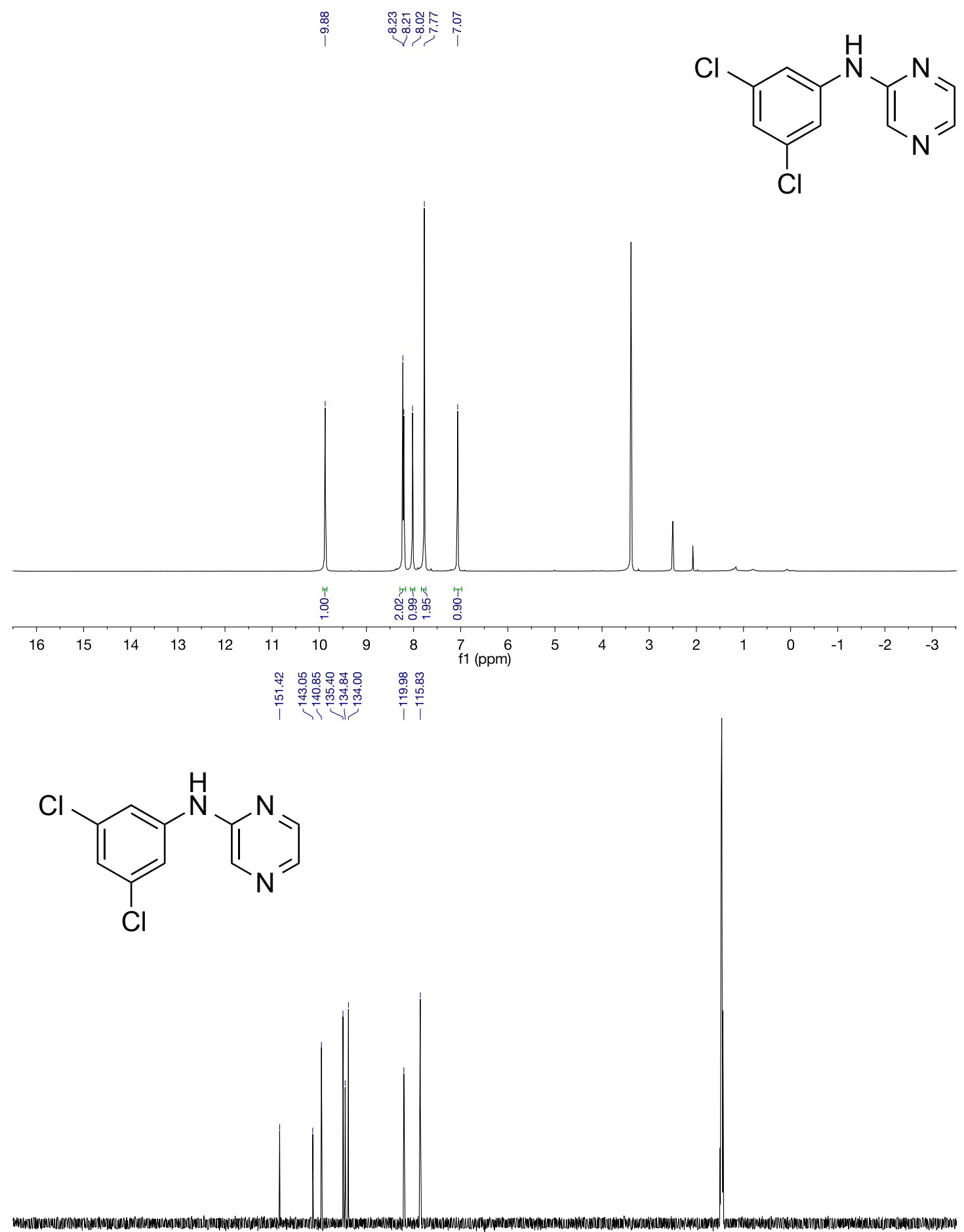

$\begin{array}{lllllllllllllllllllllll}210 & 200 & 190 & 180 & 170 & 160 & 150 & 140 & 130 & 120 & 110 & \begin{array}{c}100 \\ \mathrm{f} 1(\mathrm{ppm})\end{array} & 90 & 80 & 70 & 60 & 50 & 40 & 30 & 20 & 10 & 0 & -10\end{array}$ 
Scheme 1, Entry $3 i$
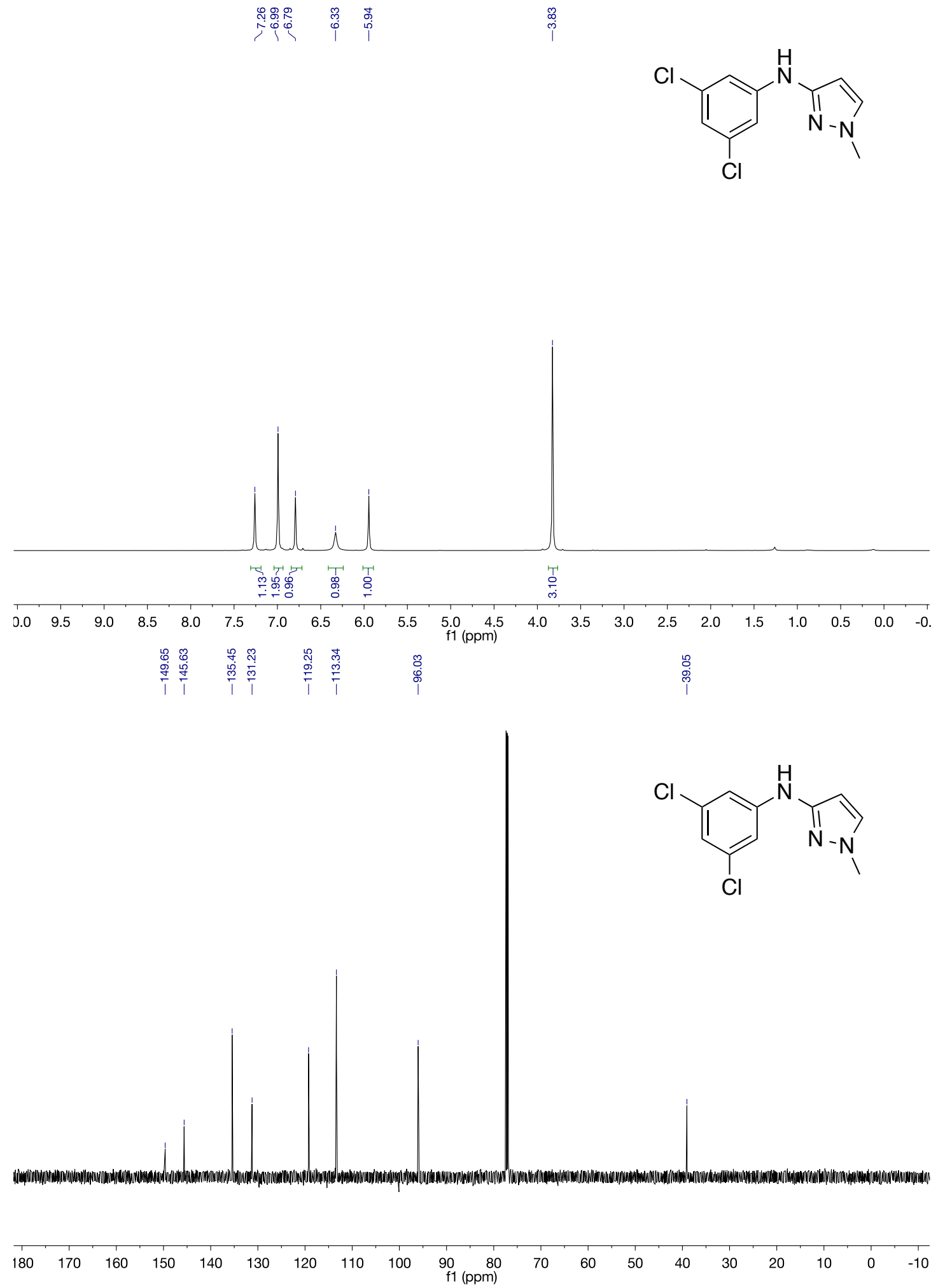
Scheme 1, Entry 3j

索

$\stackrel{\infty}{i}$<smiles>CC(=O)c1sccc1Nc1cc(Cl)cc(Cl)c1</smiles>
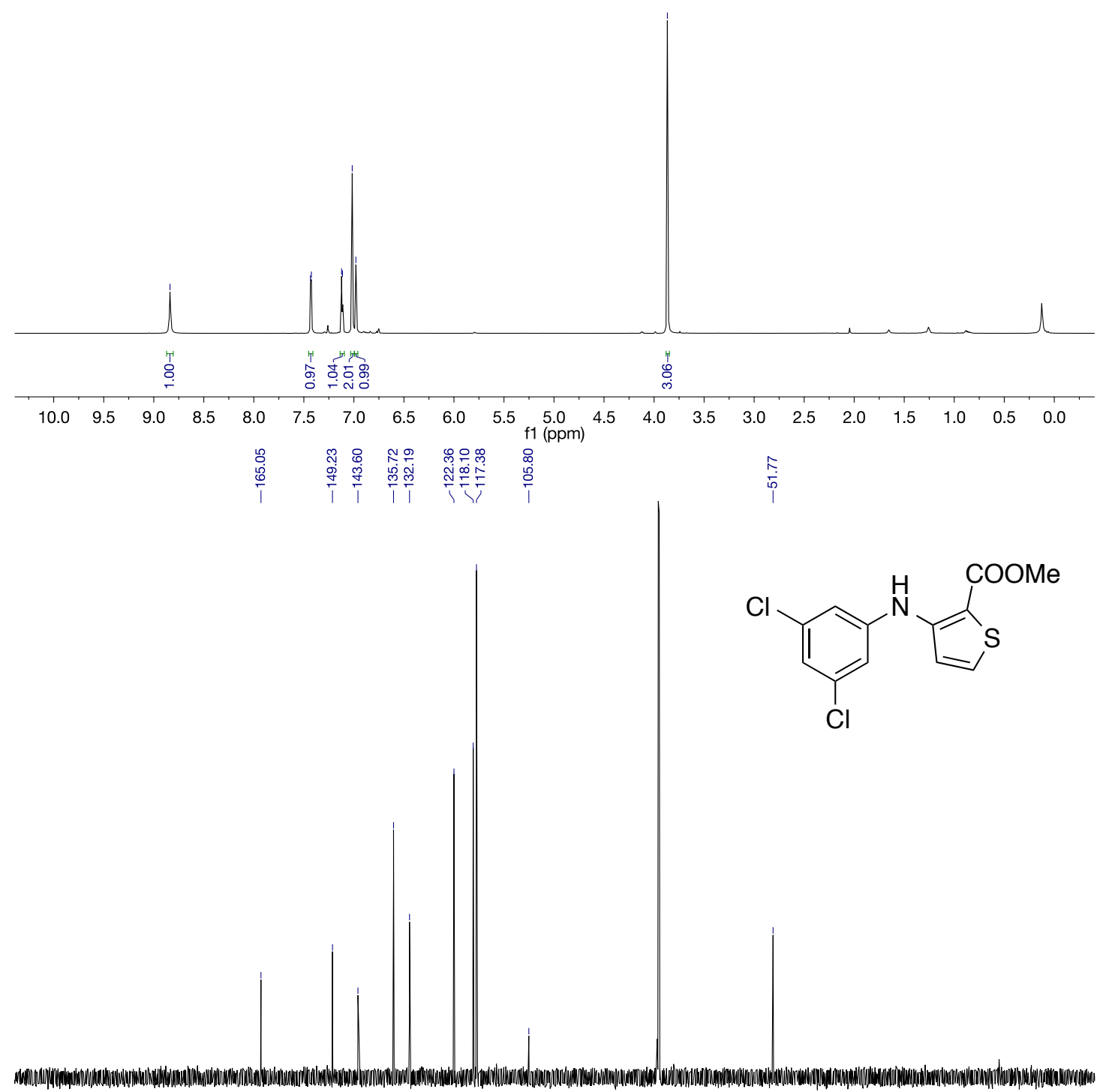

$\begin{array}{lllllllllllllllllllllll}210 & 200 & 190 & 180 & 170 & 160 & 150 & 140 & 130 & 120 & 110 & 100 & 90 & 80 & 70 & 60 & 50 & 40 & 30 & 20 & 10 & 0 & -10\end{array}$ 
Scheme 1, Entry 3k

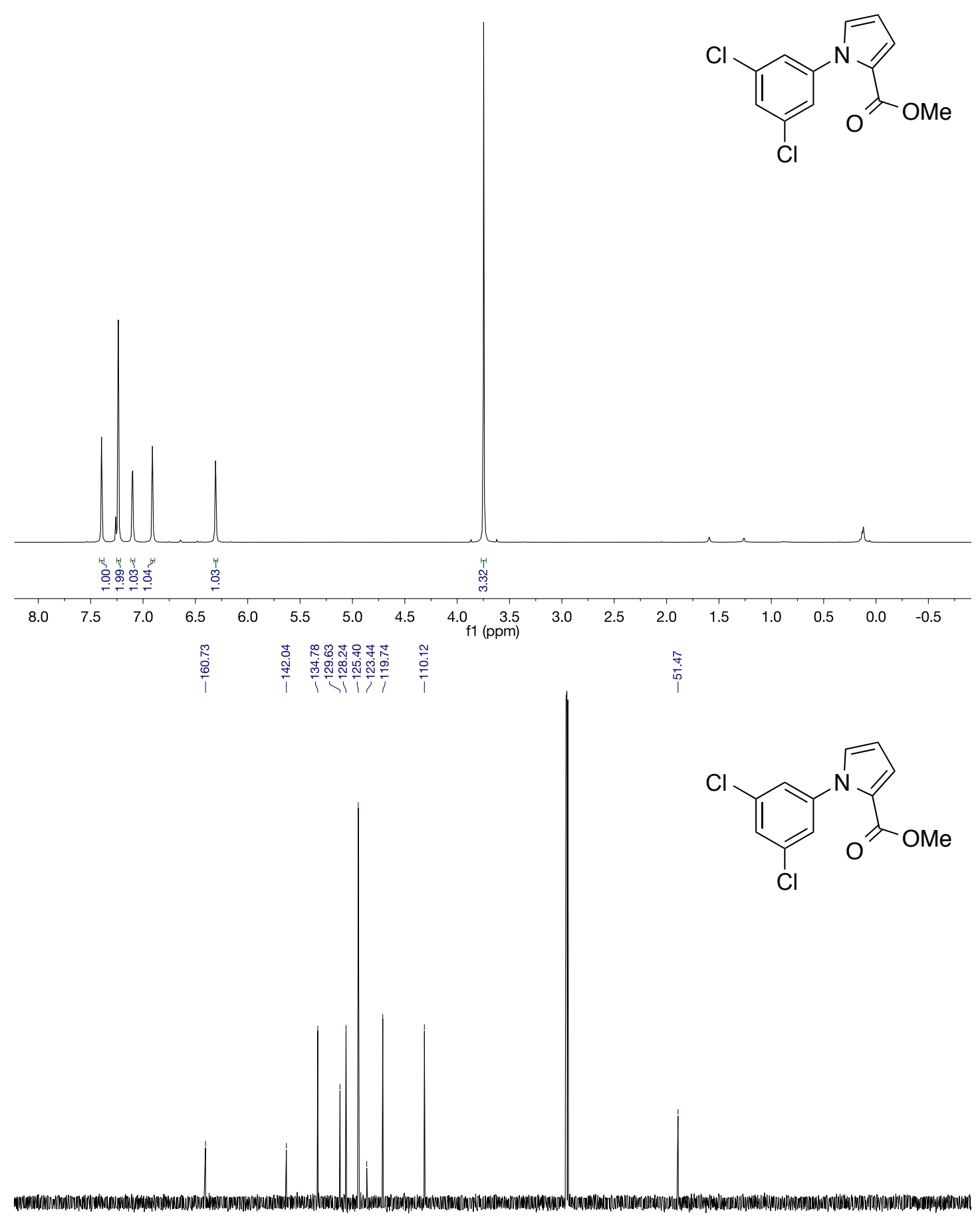

$\begin{array}{lllllllllllllllllllll}200 & 190 & 180 & 170 & 160 & 150 & 140 & 130 & 120 & 110 & \begin{array}{r}100 \\ \mathrm{f} 1\end{array}(\mathrm{ppm}) & 80 & 70 & 60 & 50 & 40 & 30 & 20 & 10 & 0 & -10\end{array}$ 
Scheme 1, Entry 31

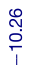

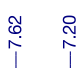

$\stackrel{\text { ֻ̊ }}{i}$<smiles>CC(=O)Nc1cc(Cl)cc(Cl)c1</smiles>

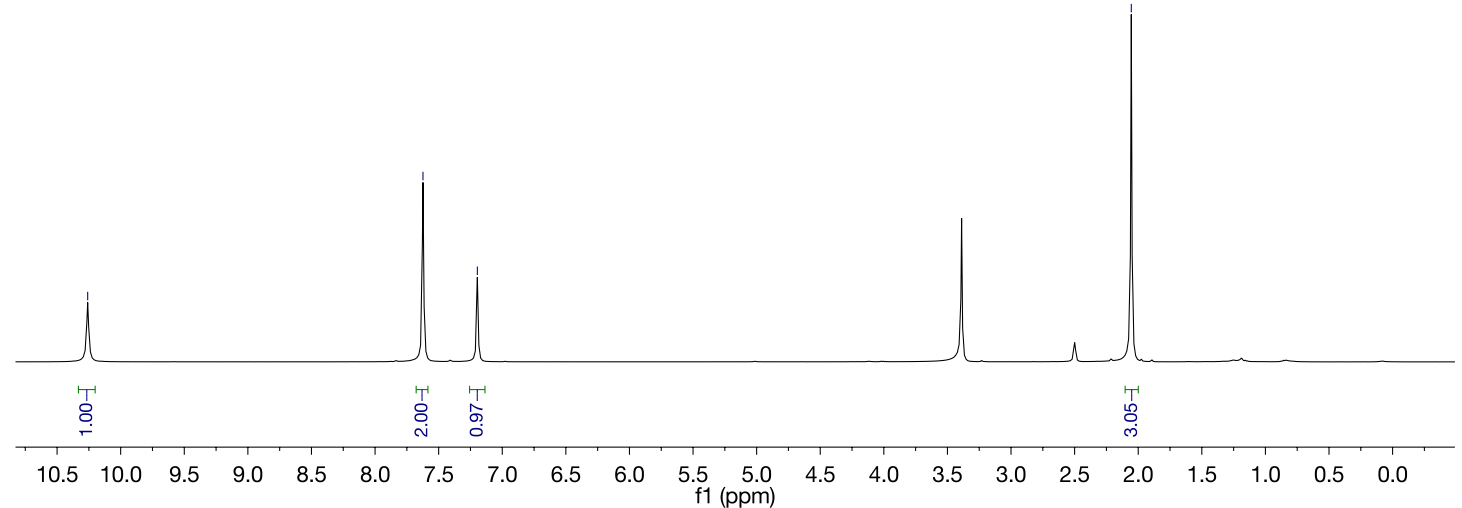<smiles>CC(=O)Nc1cc(Cl)cc(Cl)c1</smiles>

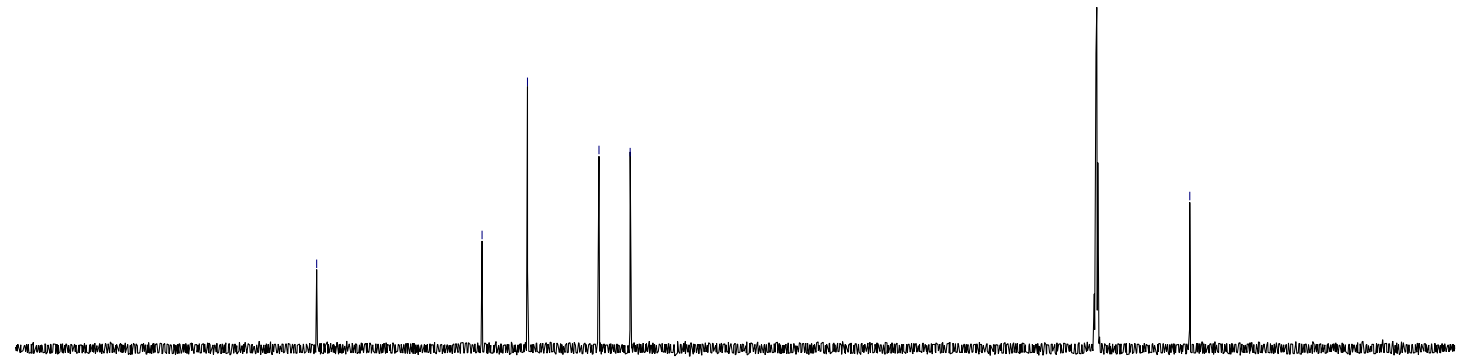

$\begin{array}{lllllllllllllllllllllll}210 & 200 & 190 & 180 & 170 & 160 & 150 & 140 & 130 & 120 & 110 & 100 & 90 & 80 & 70 & 60 & 50 & 40 & 30 & 20 & 10 & 0 & -10\end{array}$ 
Scheme 1, Entry 3m

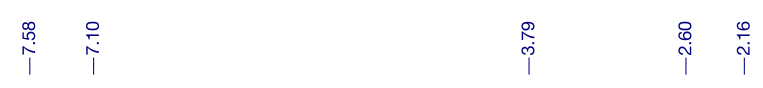<smiles>O=C1CCCN1c1cc(Cl)cc(Cl)c1</smiles>

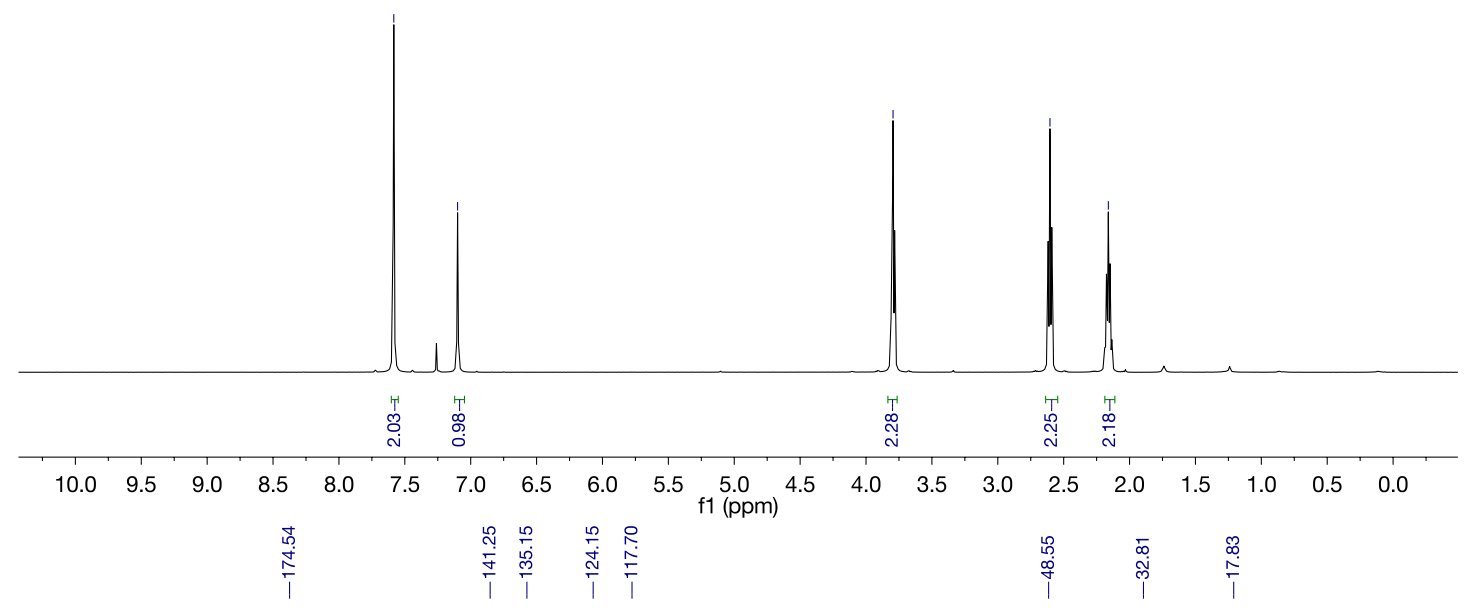<smiles>O=C1CCCN1c1cc(Cl)cc(Cl)c1</smiles>

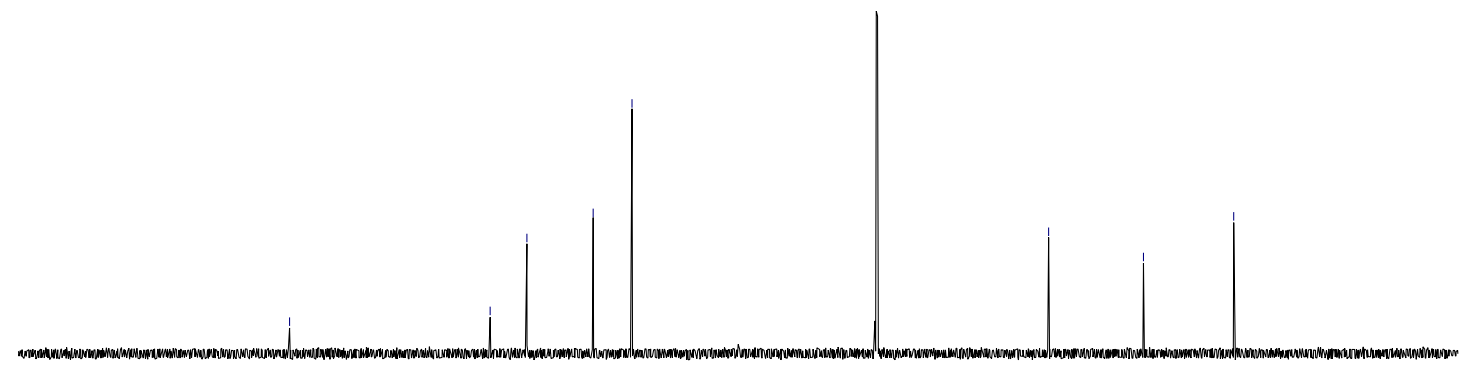

$\begin{array}{lllllllllllllllllllllll}210 & 200 & 190 & 180 & 170 & 160 & 150 & 140 & 130 & 120 & 110 & 100 & 90 & 80 & 70 & 60 & 50 & 40 & 30 & 20 & 10 & 0 & -10\end{array}$ 
Scheme 1, Entry 3n

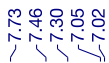
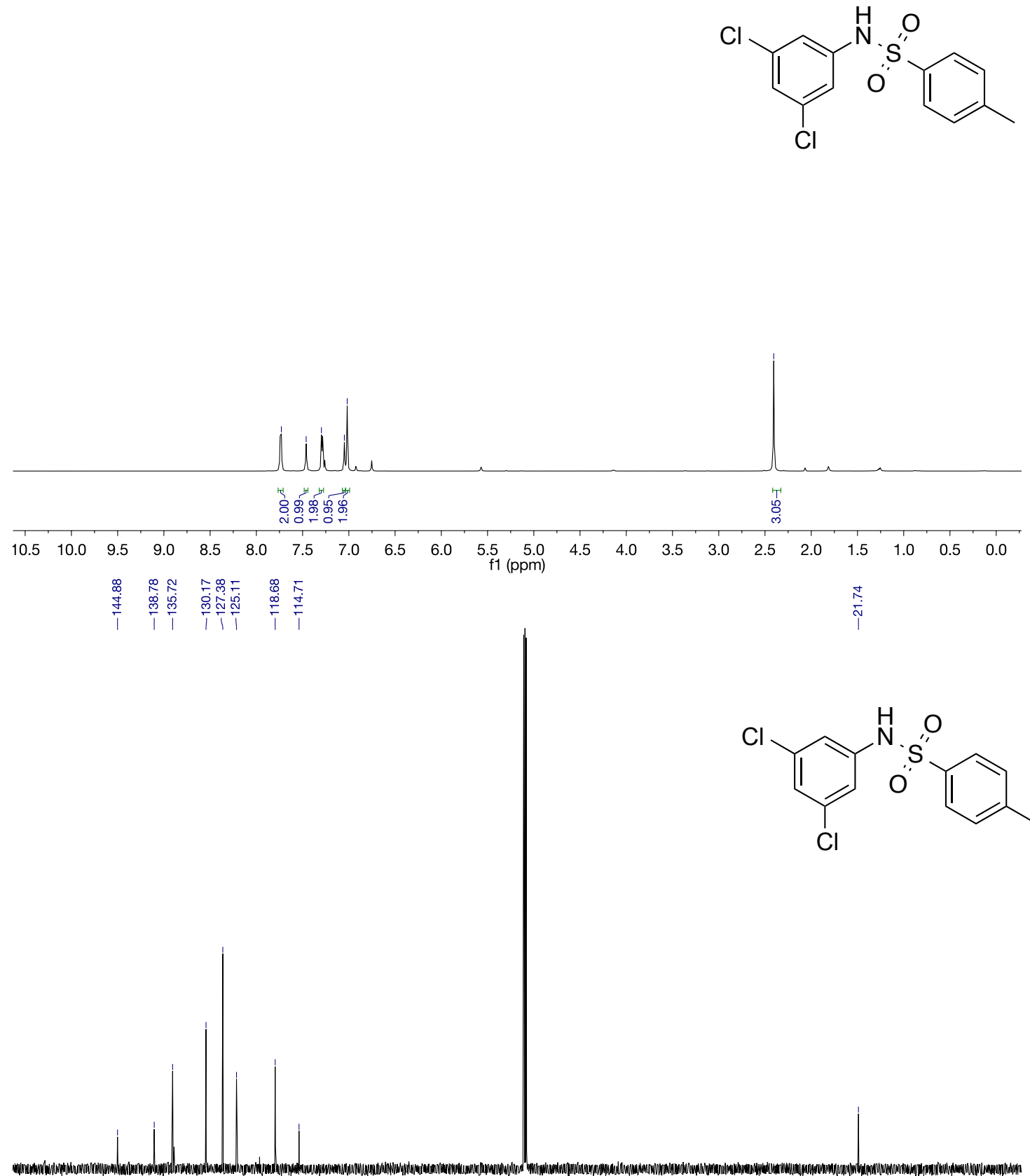

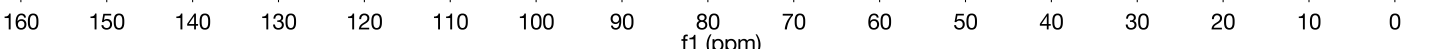


Scheme 1, Entry 30<smiles>FC(F)(F)C(F)(F)CNc1cc(Cl)cc(Cl)c1</smiles>

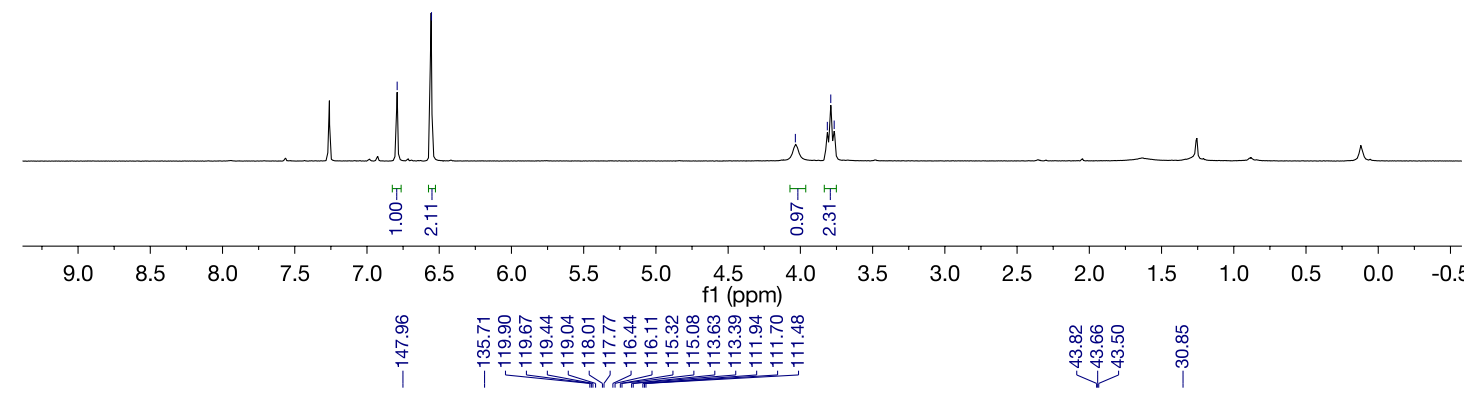<smiles>FC(F)(F)C(F)(F)CNc1cc(Cl)cc(Cl)c1</smiles> 


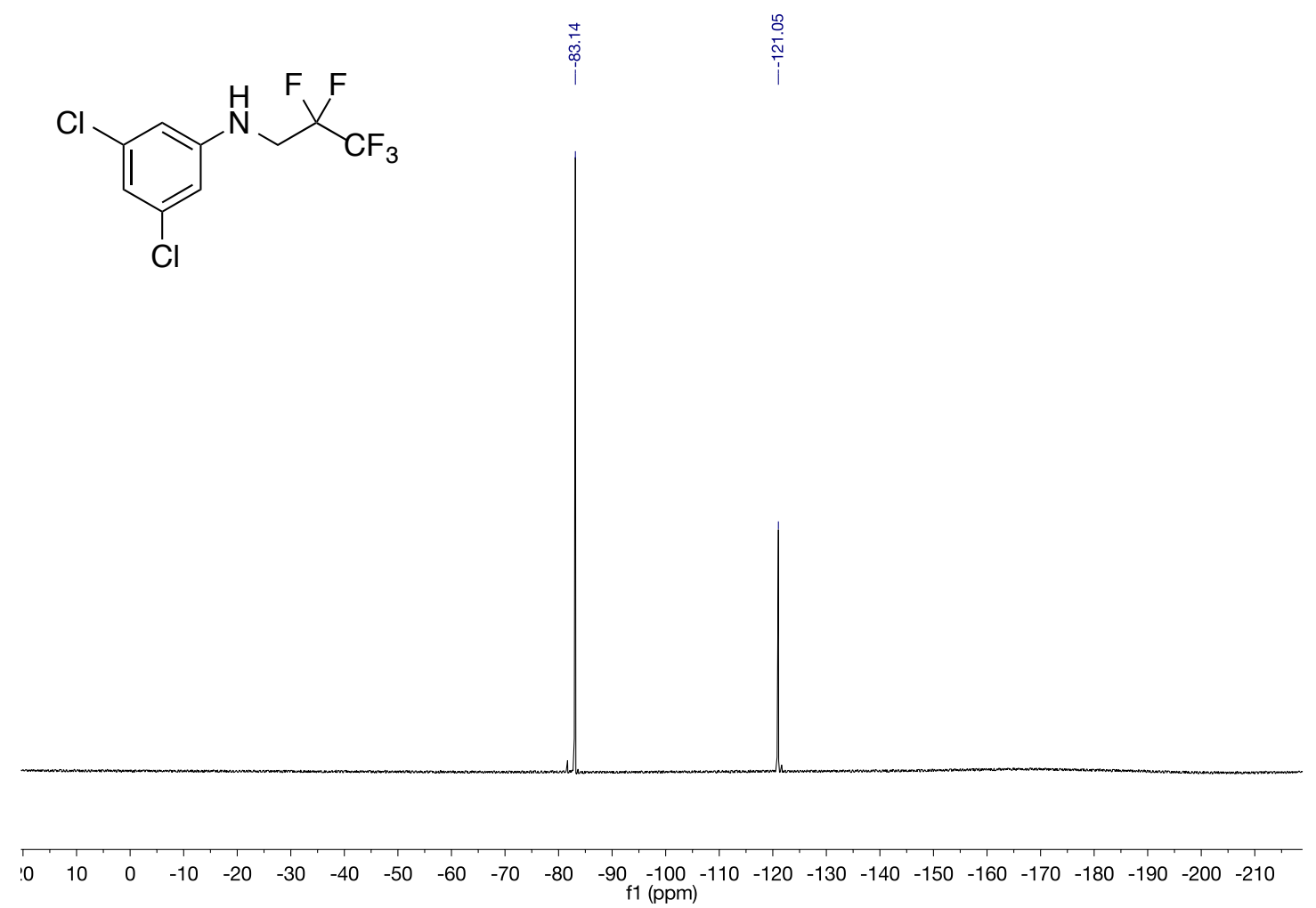


Scheme 1, Entry 3p

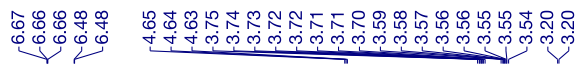<smiles>CCOC(CNc1cc(Cl)cc(Cl)c1)OCC</smiles>
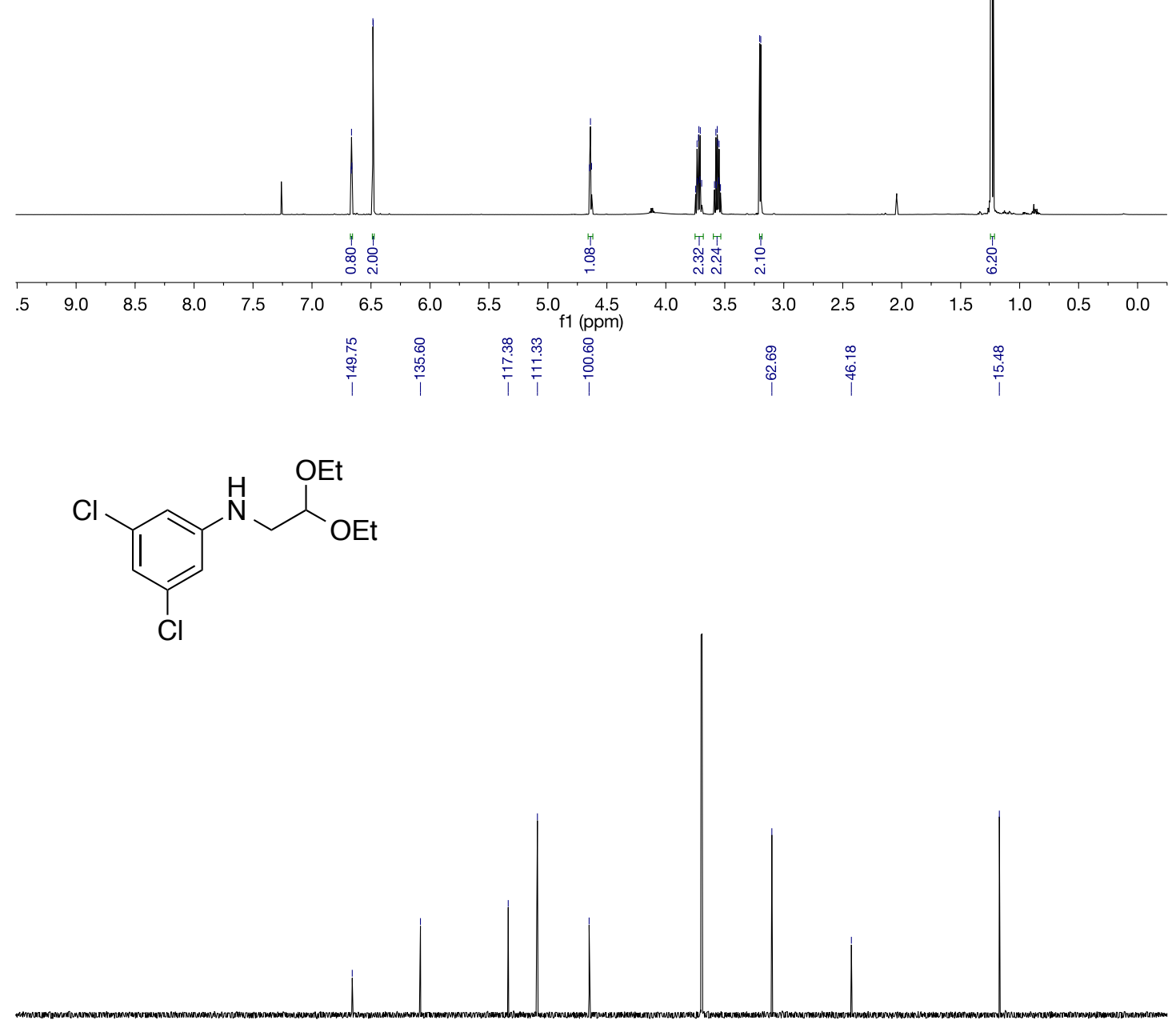

$\begin{array}{lllllllllllllllllllllll}210 & 200 & 190 & 180 & 170 & 160 & 150 & 140 & 130 & 120 & 110 & 100 & 90 & 80 & 70 & 60 & 50 & 40 & 30 & 20 & 10 & 0 & -10\end{array}$ 
Scheme 2, Entry 5a<smiles>COC(=O)c1cc(Cl)cc(N2CCCC2=O)c1</smiles>
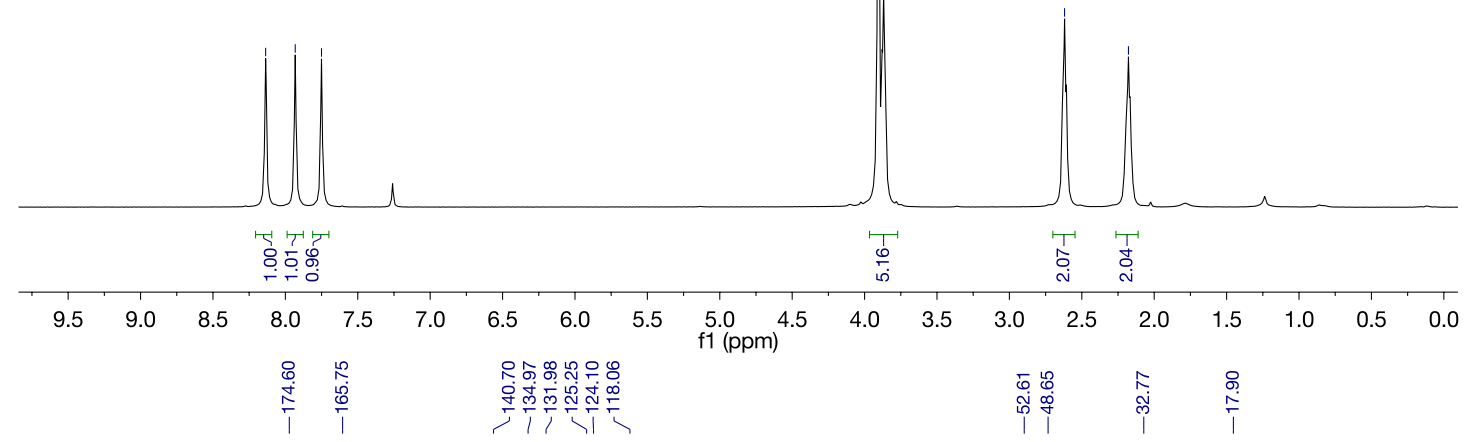<smiles>COC(=O)c1cc(Cl)cc(N2CCCC2=O)c1</smiles>

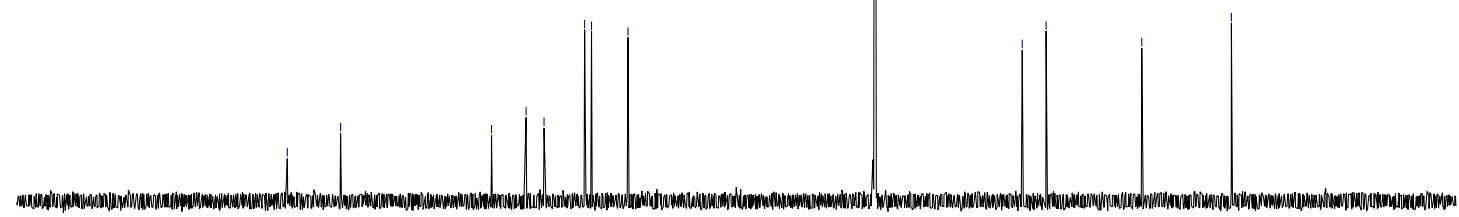

$\begin{array}{lllllllllllllllllllllll}210 & 200 & 190 & 180 & 170 & 160 & 150 & 140 & 130 & 120 & 110 \begin{array}{c}100 \\ \mathrm{f} 1(\mathrm{ppm})\end{array} & 90 & 80 & 70 & 60 & 50 & 40 & 30 & 20 & 10 & 0 & -10\end{array}$ 
Scheme 2, Entry 5b

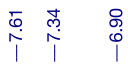

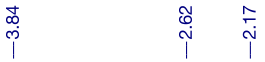<smiles>COc1cc(N2CCCC2=O)cc(C(F)(F)F)c1</smiles>

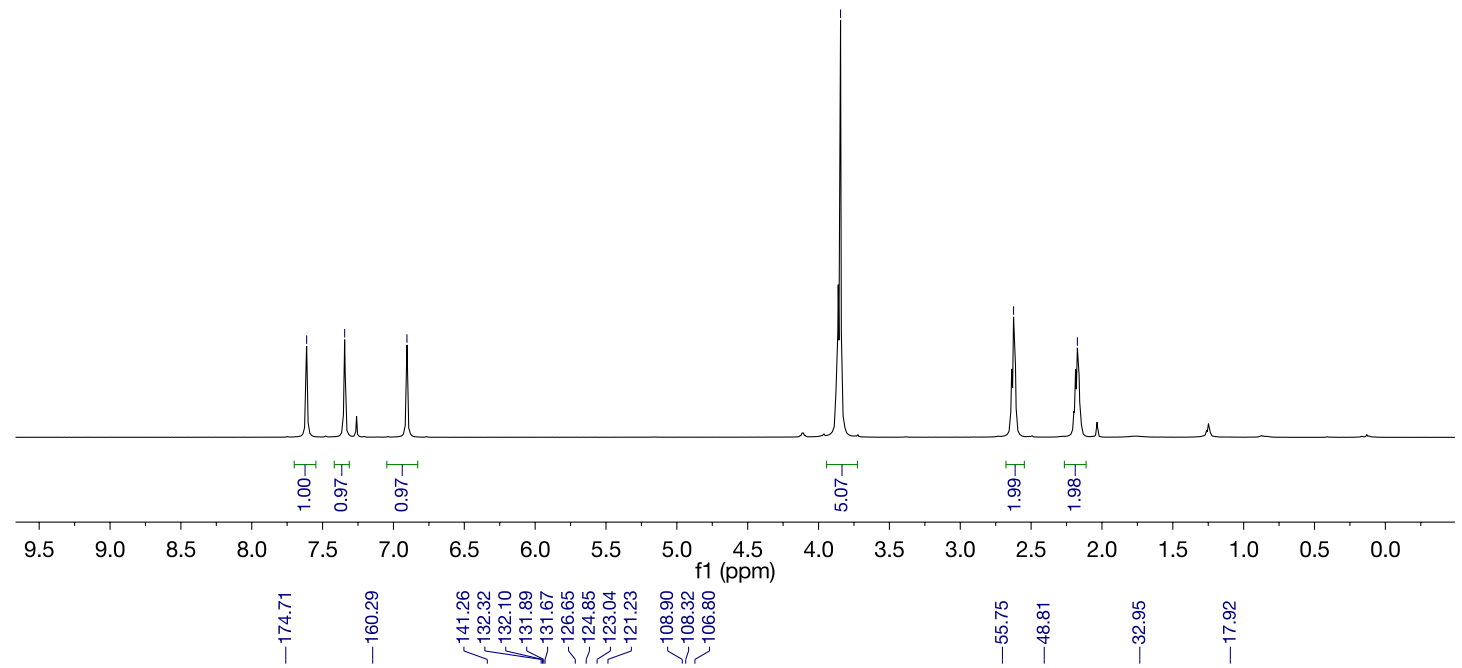<smiles>COc1cc(N2CCCC2=O)cc(C(F)(F)F)c1</smiles>

$\begin{array}{lllllllllllllllllllllll}210 & 200 & 190 & 180 & 170 & 160 & 150 & 140 & 130 & 120 & 110 & 100 & 90 & 80 & 70 & 60 & 50 & 40 & 30 & 20 & 10 & 0 & -10\end{array}$ 


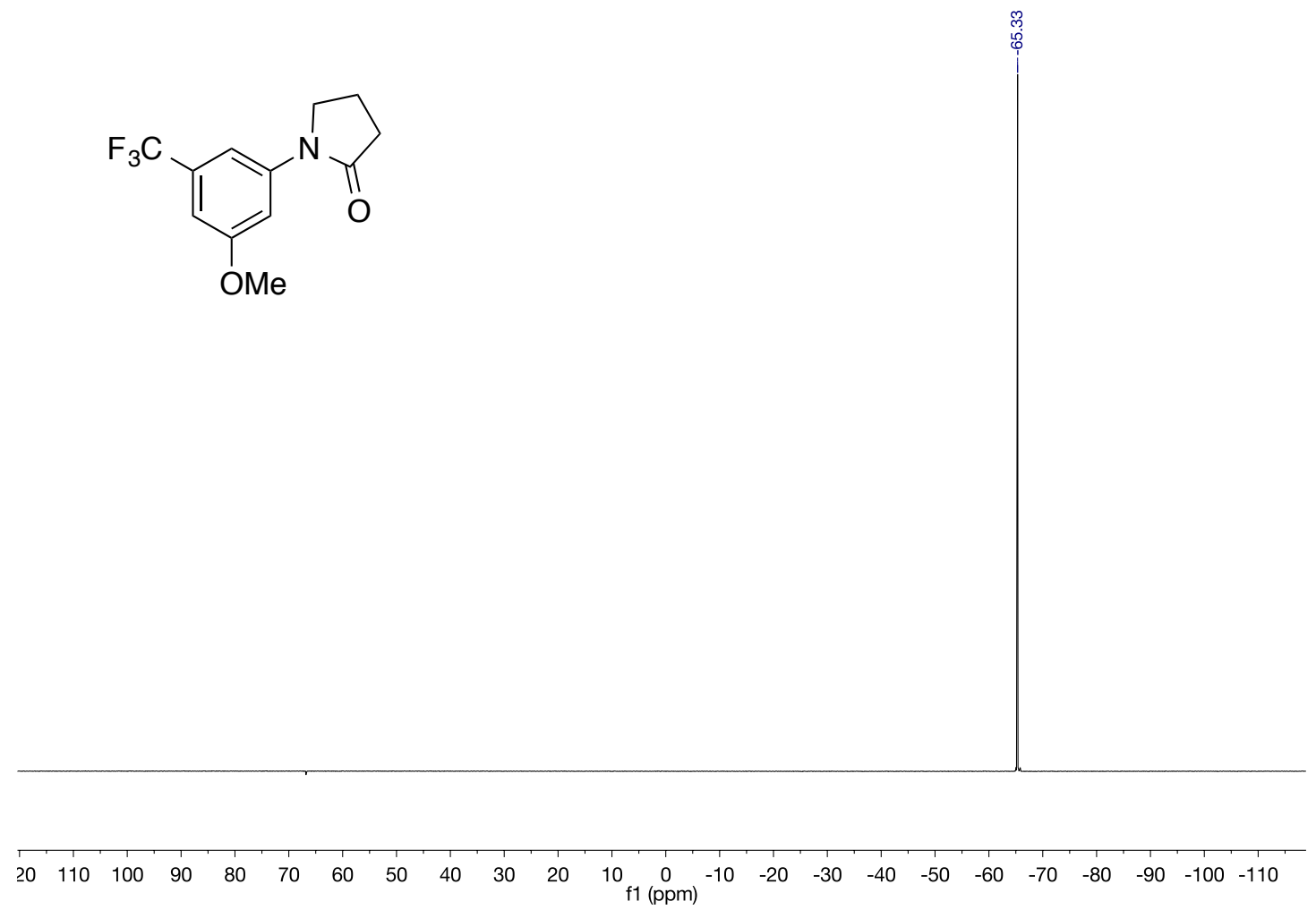


Scheme 2, Entry 5c

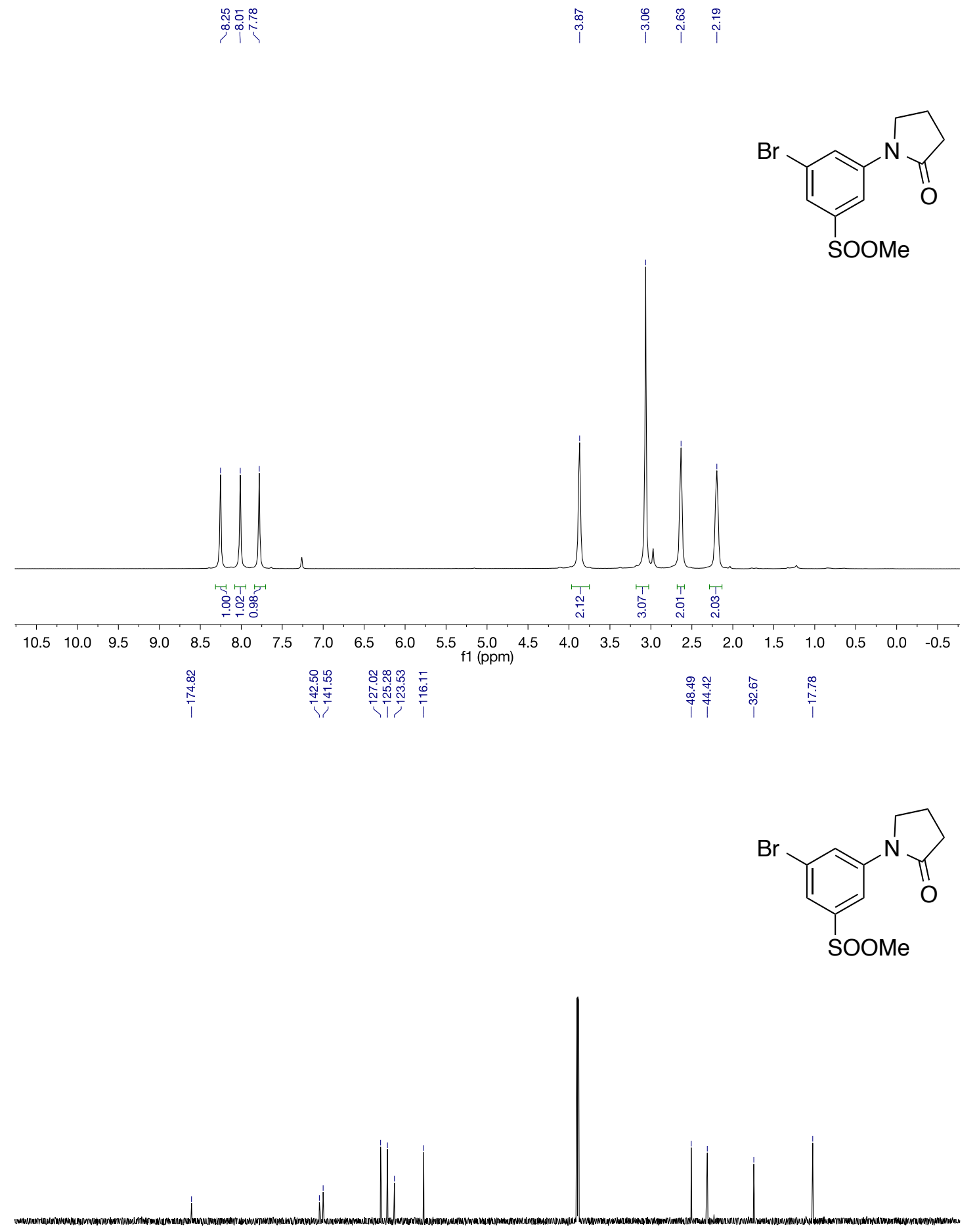

$\begin{array}{lllllllllllllllllllllll}210 & 200 & 190 & 180 & 170 & 160 & 150 & 140 & 130 & 120 & 110 & 100 & 90 & 80 & 70 & 60 & 50 & 40 & 30 & 20 & 10 & 0 & -10\end{array}$ 
Scheme 2, Entry 5d

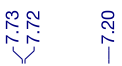

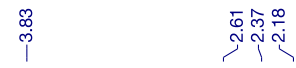

$\overbrace{\mathrm{CN}}^{\mathrm{O}}$
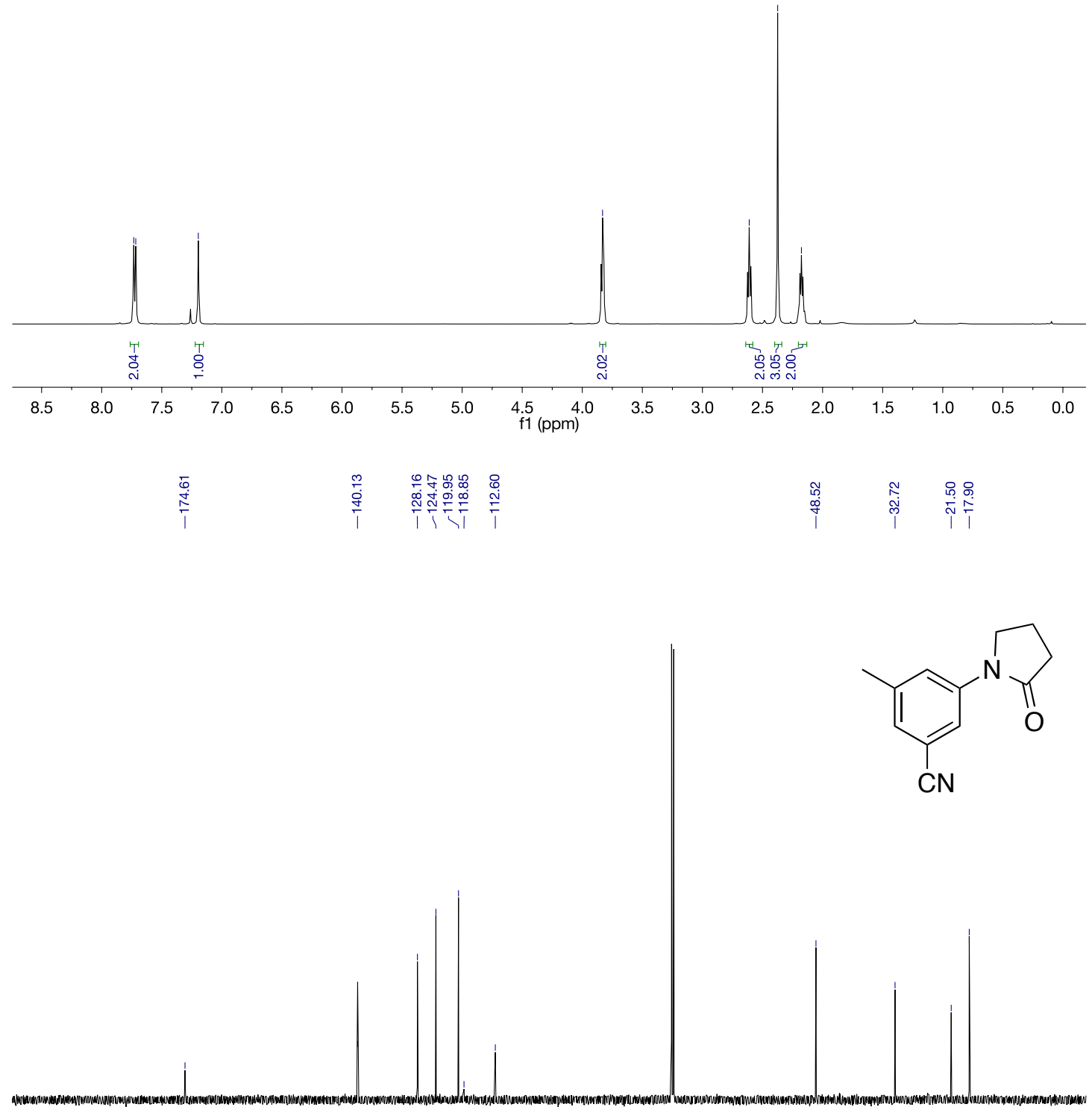

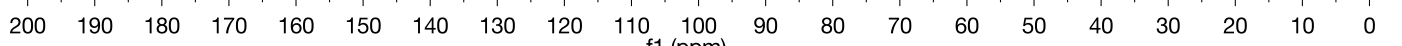


Scheme 2, Entry 5e

品

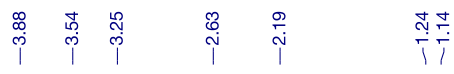<smiles>CCOC(=O)c1cc(N2CCCC2=O)cc(C(F)(F)F)c1</smiles>
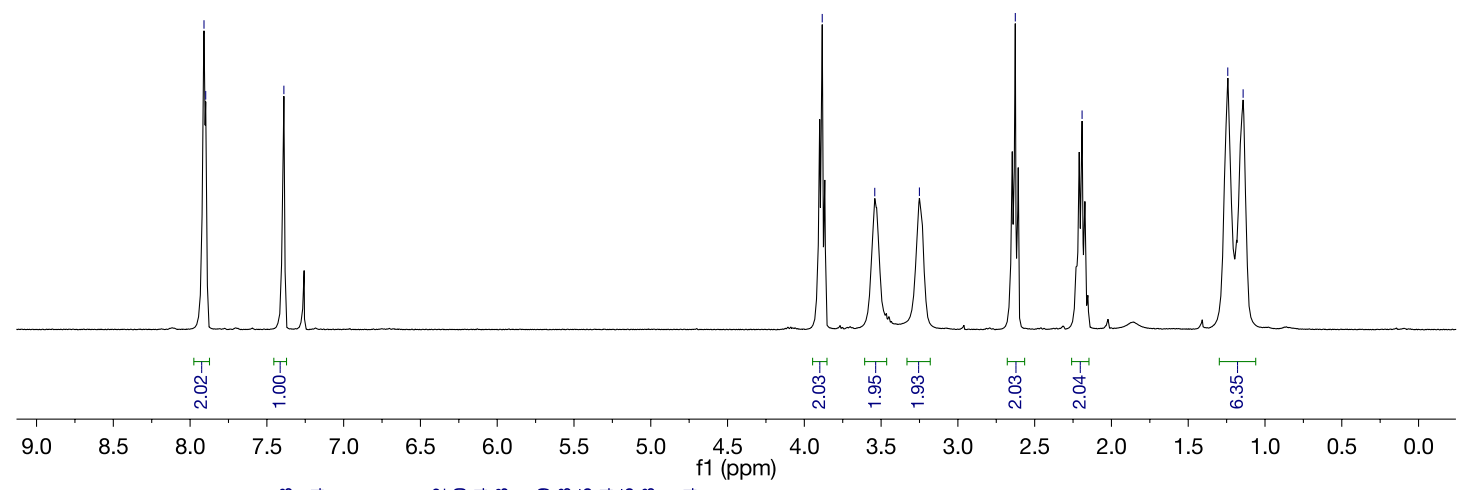

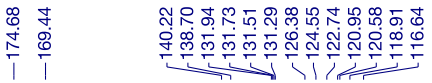

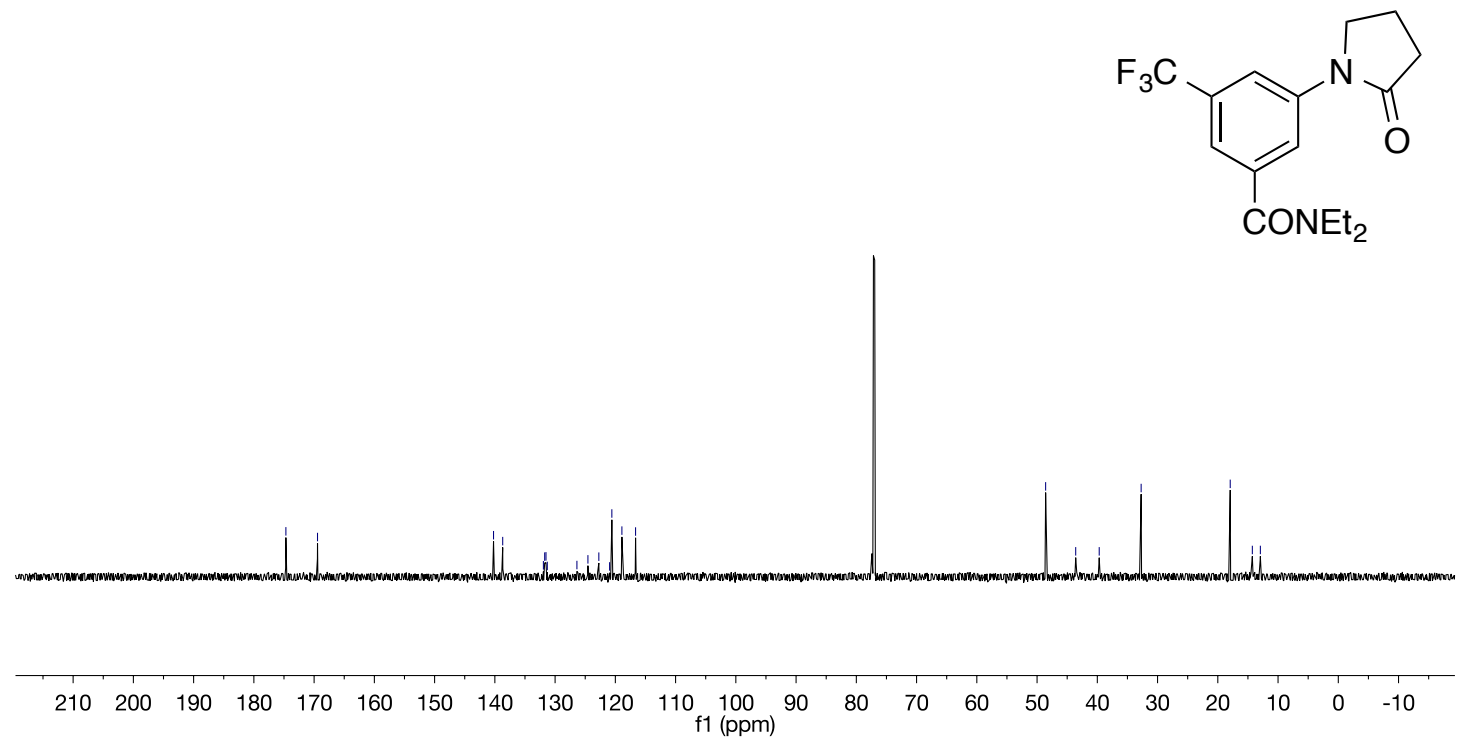


<smiles>CCOC(=O)c1cc(N2CCCC2=O)cc(C(F)(F)F)c1</smiles>

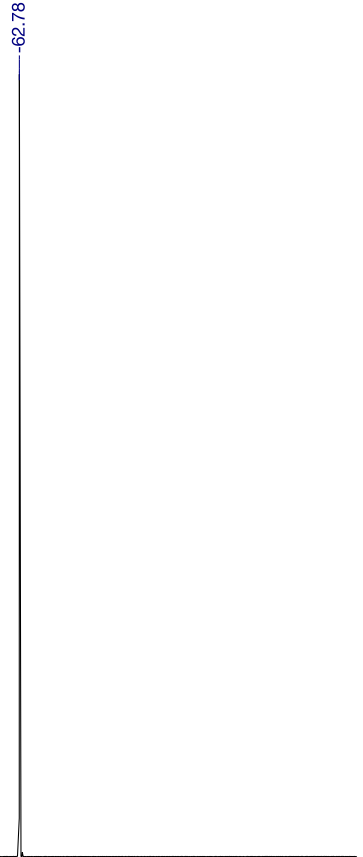

\begin{tabular}{lllllllllllllllllllllll}
\hline 0 & 110 & 100 & 90 & 80 & 70 & 60 & 50 & 40 & 30 & 20 & $10 \underset{\mathrm{f} 1(\mathrm{ppm})}{0}$ & -10 & -20 & -30 & -40 & -50 & -60 & -70 & -80 & -90 & -100 & -110
\end{tabular} 
Scheme 2, Entry $5 f$

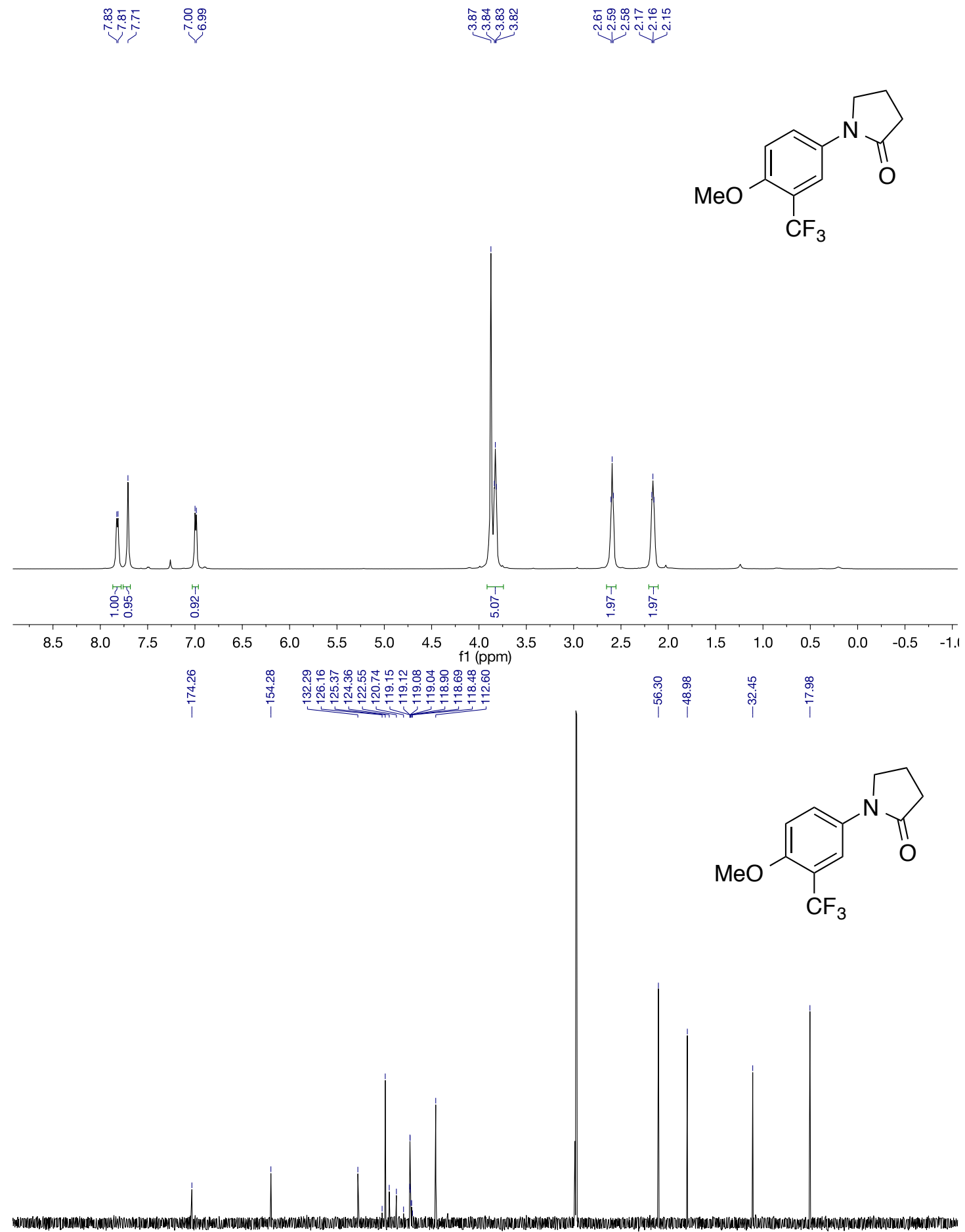

$\begin{array}{lllllllllllllllllllllll}210 & 200 & 190 & 180 & 170 & 160 & 150 & 140 & 130 & 120 & 110 \begin{array}{c}100 \\ \mathrm{f} 1(\mathrm{ppm})\end{array} & 90 & 80 & 70 & 60 & 50 & 40 & 30 & 20 & 10 & 0 & -10\end{array}$ 
<smiles>COc1ccc(N2CCCC2=O)cc1C(F)(F)F</smiles>

$\begin{array}{lllllllllllllllllllllllll}20 & 110 & 100 & 90 & 80 & 70 & 60 & 50 & 40 & 30 & 20 & 10 & 0 & 0 & -10 & -20 & -30 & -40 & -50 & -60 & -70 & -80 & -90 & -100 & -110\end{array}$ 
Scheme 2, Entry 5g

$\underbrace{100}$

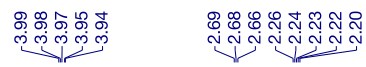

$\mathrm{MeOOCOC}$

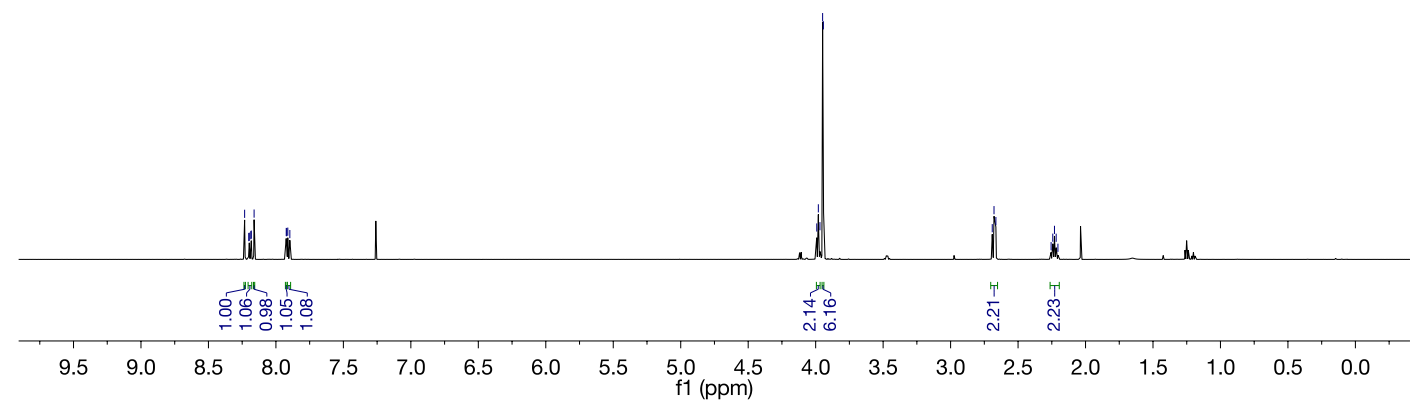

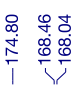

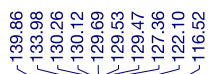

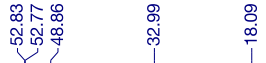

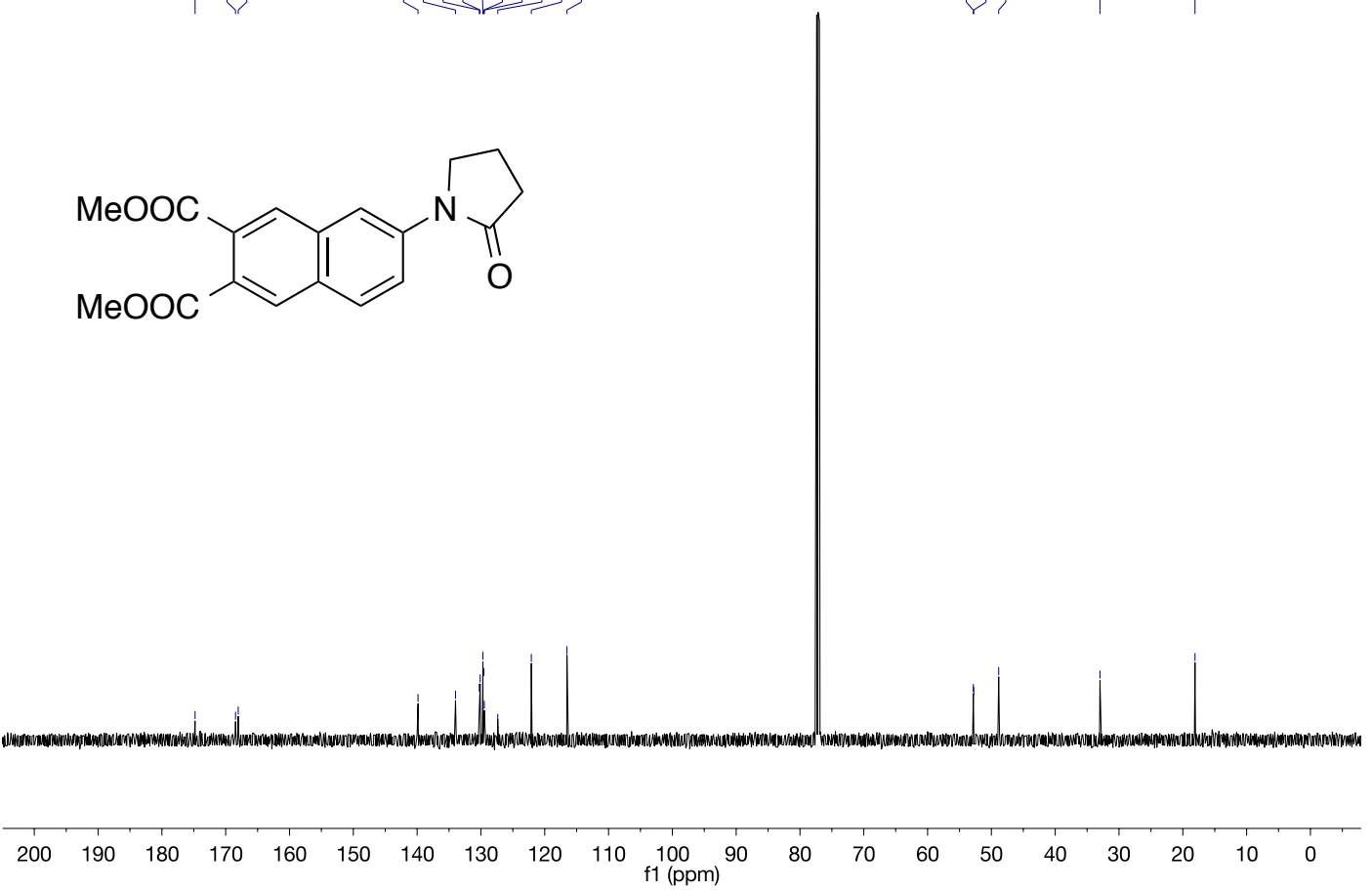


Scheme 2, Entry 5h

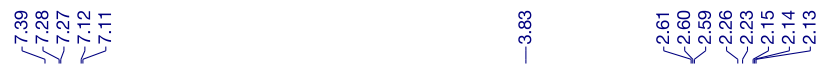<smiles>Cc1ccc(N2CCCC2=O)cc1C</smiles>
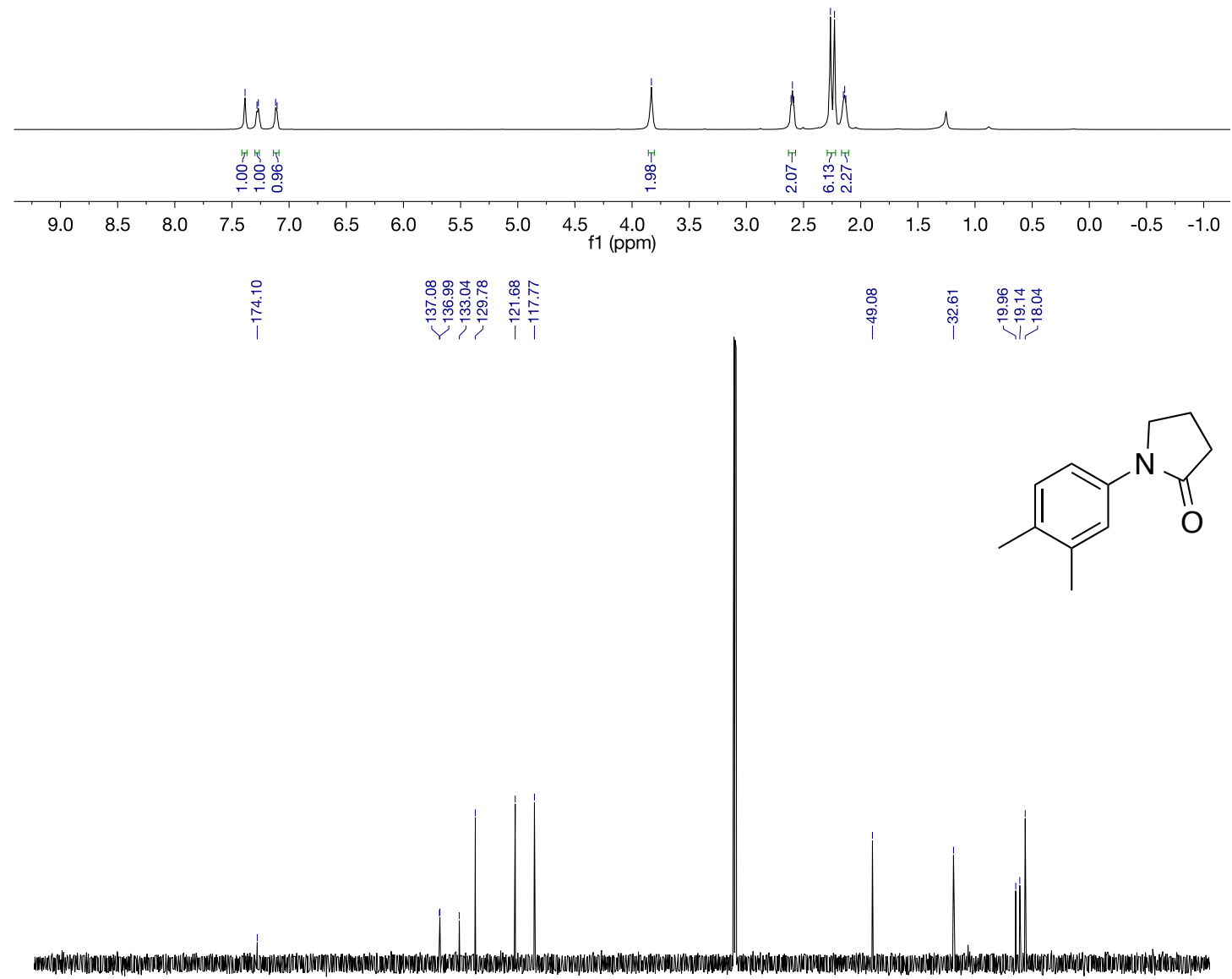

$\begin{array}{lllllllllllllllllllllll}210 & 200 & 190 & 180 & 170 & 160 & 150 & 140 & 130 & 120 & 110 & 100 & 90 & 80 & 70 & 60 & 50 & 40 & 30 & 20 & 10 & 0 & -10\end{array}$ 
Scheme 2, Entry $5 i$

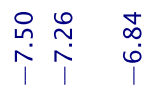

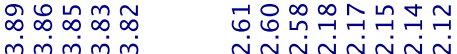<smiles>COc1ccc(N2CCCC2=O)cc1OC</smiles>

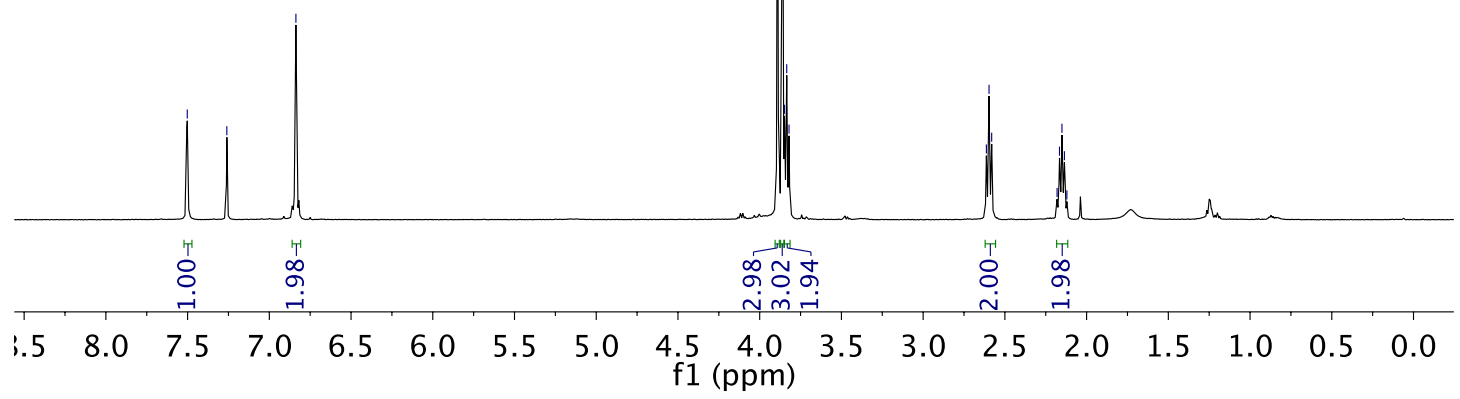

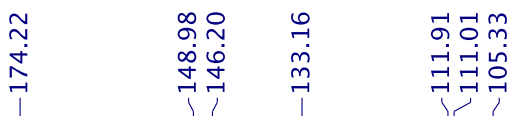

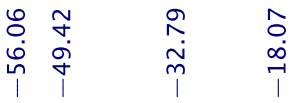<smiles>COc1ccc(N2CCCC2=O)cc1OC</smiles>

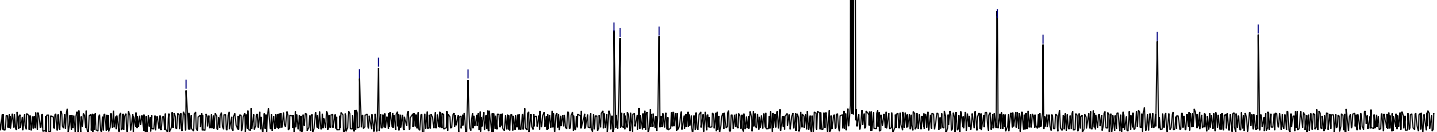

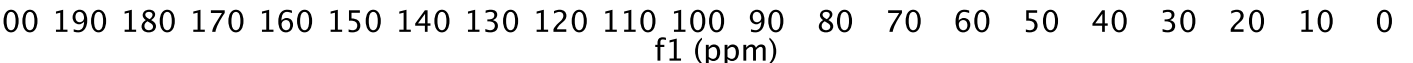


Scheme 2, Entry 5j

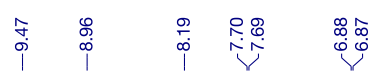<smiles>Cc1ncc(Nc2ccc(Cl)cn2)cn1</smiles>
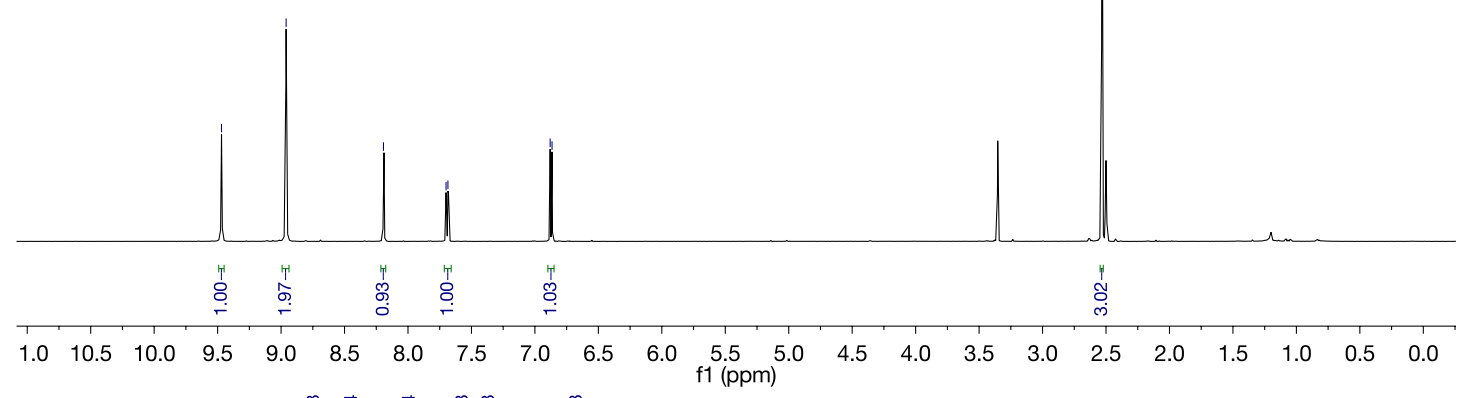
।<smiles>Cc1ncc(Nc2ccc(Cl)cn2)cn1</smiles>

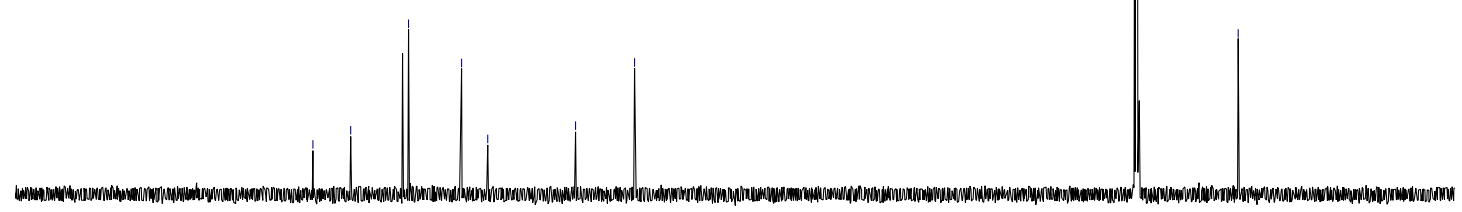

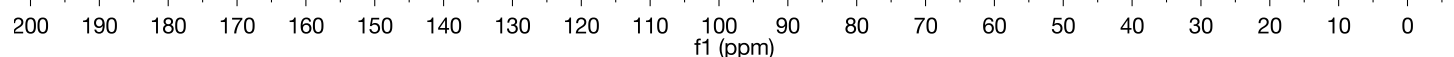

